\title{
«Des étrangers et des gens du dehors ». Ou quand les Johnnies ne s'appelaient pas Petit Jean
}

'Strangers and foreigners', or when the 'Johnnies' were not called 'Petit Jean'

\section{Estelle Boudillet}

\section{QpenEdition}

\section{Journals}

Édition électronique

URL : http://journals.openedition.org//bl/425

DOI : $10.4000 / \mathrm{lbl} .425$

ISSN : $2727-9383$

Éditeur

Université de Bretagne Occidentale - UBO

\section{Édition imprimée}

Date de publication : 1 mars 2016

Pagination : 45-88

ISBN : 979-10-92331-24-0

ISSN : $1270-2412$

Référence électronique

Estelle Boudillet, « «Des étrangers et des gens du dehors ». Ou quand les Johnnies ne s'appelaient pas Petit Jean », La Bretagne Linguistique [En ligne], 20 | 2016, mis en ligne le 04 mars 2020, consulté le 01 octobre 2020. URL : http://journals.openedition.org//bl/425 ; DOI : https://doi.org/10.4000//bl.425

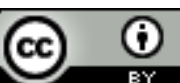

La Bretagne Linguistique est mise à disposition selon les termes de la Licence Creative Commons Attribution 4.0 International. 


\author{
Estelle BOUDILLET*
}

\title{
"Des étrangers et des gens du dehors". Ou quand les Johnnies ne s'appelaient pas Petit Jean ${ }^{1}$
}

\author{
À la mémoire de Sébastien Prigent (1921-2013). \\ À Yvon Corre, qui m'a mise sur la voie d'un autre Y.C.
}

This nameless mode of naming the unnameable is quite good ${ }^{2}$.

"I don't want thumbscrews or the rack, but there always seems to be something of Johnny Foreigner about the Catholics ${ }^{3}$. »

* Doctorante en celtique, CRBC (EA 4451), UBO.

1. Le présent article s'inscrit dans le prolongement de mon mémoire de Master 2 : «Tribulations de "Johnny" lors de ses premières années. Apparition et diffusion du vocable "Johnny" pour désigner les marchands d'oignons léonards en Grande-Bretagne et Bretagne de 1877 à 1939 », Brest, UBO, 2013. Constituant un nouveau jalon dans un projet de recherche au long cours, il vise à asseoir plus fermement les prémisses sur lesquelles repose ma tentative de reconstitution de l'histoire et de mise en question du sens du surnom des marchands d'oignons léonards entre le dernier quart du XIXe siècle et la Seconde Guerre mondiale (voir les balises chronologiques données en annexe).

2. "Cette manière anonyme de nommer l'innommable convient assez bien. " Mary Wollstonecraft Shelley, 1823, dans Betty T. Bennett, Selected Letters of Mary Wollstonecraft Shelley, Baltimore, Johns Hopkins University Press, 1995, p. 129. Sauf mention contraire, les traductions de l'anglais sont les miennes.

3. « Je ne réclame pas le supplice des poucettes ou du chevalet, mais ces catholiques me font toujours un peu l'effet de métèques. » (Réplique prononcée par le personnage de Lord Grantham, s'adressant à l'Archevêque d'York - second primat de l'Église anglicane -, lors d'une scène censée se dérouler au plus fort de la Guerre d'indépendance irlandaise de 1919-1921. Julian Fellowes, Downton Abbey, Série 3, épisode 4, 7 oct. 2012 pour la 1 re diffusion à la télévision britannique.) 
rendre les marchands d'oignons léonards ${ }^{4}$ pour sujet aujourd'hui en Bretagne appelle automatiquement l'utilisation de la désignation JOHNNY ${ }^{5}$. Mis en exergue en tant que mot-clef d'intitulés («Henry Ollivier, premier Johnny ${ }^{6}$ ? », « L'Histoire critique des Johnnies $\left.{ }^{7} »\right)^{8}$, ce pérégrinisme d'origine populaire s'est vu hissé au cours de la dernière décennie au rang de mot-vedette placé en tête de notice dans des ouvrages

4. Afin de minimiser le risque de circularité inhérent à tout discours portant sur des faits discursifs, je réserverai mon emploi du mot JOHNNY (noté en majuscules quand il s'agira du signe-type subsumant toutes ses actualisations potentielles) à sa fonction autonymique. Lorsque je renverrai non au mot lui-même mais au référent auquel cette dénomination est aujourd'hui communément associée en Bretagne, j'aurai alors recours à des formules descriptives comme " marchand(s) d'oignons léonard(s)».

5. J'ai relevé une exception notable chez Joël Cornette, qui évoque le démarrage du commerce trans-Manche des oignons sans caractériser les instigateurs de ce négoce par quelque désignation collective que ce soit (Histoire de la Bretagne et des Bretons, t. 2, Paris, éditions du Seuil, 2008 [2005], p. 254).

6. Dany Guillou-Beuzit, «Henri Ollivier, premier Johnny ? ", Langues de l'Histoire, langues de la vie : mélanges offerts à Fañch Roudaut, Brest, UBO, 2005, p. 476-483.

7. Titre d'une communication donnée le 4 juin 2013 par Jean Lefranc dans le cadre de conférences organisées à l'UBO par la Société d'Études de Brest et du Léon.

8. C'est hors de Bretagne que l'efficacité dénotative et la force connotative du vocable JOHNNY ont été mises en œuvre pour la première fois afin d'identifier et désigner une étude scientifique - la première, à ma connaissance, à s'être penchée spécifiquement sur l'activité des marchands d'oignons léonards. L'article «Les Johnnies du Nord Finistère », paru à Paris en 1957 dans un périodique étroitement spécialisé (Le Bulletin des Engrais), fut repris peu après, sous un intitulé rallongé à des fins d'explicitation descriptive (tandis que le mot-thème lui-même se trouvait mystérieusement raccourci d'un de ses $<\mathrm{n}>$ ), dans une revue au champ disciplinaire plus large mais à l'objet d'étude plus circonscrit spatialement (André MEYNIER, «Les Johnies du Nord Finistère en Grande Bretagne ", Norois, Revue Géographique de l'Ouest et des Pays de l'Atlantique Nord, $\mathrm{n}^{\circ} 18$, Instituts de Géographie des Facultés des Lettres de Caen, Poitiers, Rennes/ CNRS, Poitiers, avril-juin 1958, p. 231).

Bien que le mot JOHNNY n'intervienne pas dans les titres de ses travaux, c'est l'ethnomusicologue Claudie Marcel-Dubois qui avait ouvert la voie quelques années auparavant en apprivoisant et s'appropriant la désignation au cours d'une enquête de terrain menée à Roscoff et l'île de Batz entre 1951 et 1953. Son usage intensif de l'appellation - 29 occurrences dans un compte-rendu de 2 pages («Les Marchands d'oignons du Pays de Léon : Leur Répertoire musical et leurs campagnes en Angleterre », Arts et Traditions Populaires, $\mathrm{n}^{\circ}$ 4, oct-déc. 1954, p. 337-339) - s’illustre par la très grande souplesse avec laquelle la scientifique adapte le mot à des rôles grammaticaux variés, e.g. : « un johnny de Cléder» (nom commun singulier), « les premiers johnnies » (nom commun pluriel), « le répertoire musical johnny » (adjectif masculin), « l'affaire johnnie » (adjectif féminin), etc. (Voir également « Musique populaire vocale de l'̂̂le de Batz », Arts et Traditions Populaires, n³ juil.-sept. 1954, p. 193-250.) 
encyclopédiques de référence ${ }^{9}$ et semble désormais faire partie intégrante du lexique partagé de la communauté discursive bretonne ${ }^{10}$.

Unanimement adopté et pleinement intégré ${ }^{11}$, l'outil désignatif

9. Le Dictionnaire du patrimoine breton et le Dictionnaire d'histoire de Bretagne présentent respectivement les adresses « Johnies » et « Johnnies » (Alain CroIX et JeanYves Veillard (dir.), Dictionnaire du patrimoine breton, Rennes, éditions Apogée, 2e éd., 2001, p. 523-524 ; Jean-Christophe CASSARD, A. CroiX, Jean-René LE QUÉAU et J.-Y. Veillard (dir.), Dictionnaire d'histoire de Bretagne, Morlaix, Skol Vreizh, 2008, p. 413-414.)

10. Parallèlement, l'inclusion d'une entrée «Johnny» dans un glossaire des locutions françaises de Basse-Bretagne sanctionne la reconnaissance par la communauté scientifique bretonne de l'intégration de cette unité lexicale dans la pratique linguistique commune ou majoritaire de l'ensemble des locuteurs bas-bretons (cf. Jean LE DÛ, Du Café vous aurez? Quand les Bretons parlent français, Brest, Emgleo Breiz, nouv. éd., 2011 [2002], p. 161).

Dans le même temps, le pluriel «Johnnies » a pénétré les plus hautes sphères du vocabulaire juridico-administratif français lors de la parution au Journal officiel de la reconnaissance en "Appellation d'Origine Contrôlée » de l'oignon rosé (désormais labellisé « oignon de Roscoff »), où 13 occurrences de cette forme viennent conforter l'argumentaire patrimonial nécessaire à l'obtention de la certification («Décret $\mathrm{n}^{\circ}$ 2009-1268 du 19 octobre 2009 relatif à l'appellation d'origine contrôlée "oignon de Roscoff" ", Journal Officiel de la République Française, n 0245, 22 octobre 2009, en ligne : http://www.legifrance.gouv.fr/eli/decret/2009/10/19/AGRT0917334D/jo/ texte). Cette institutionnalisation a ensuite été confirmée et transcendée, en passant du niveau national à l'échelle européenne, lors de l'enregistrement de la demande de dénomination "Appellation d'Origine Protégée» («Publication d'une demande en application de l'article 6, paragraphe 2, du règlement (CE) $n^{\circ}$ 510/2006 du Conseil relatif à la protection des indications géographiques et des appellations d'origine des produits agricoles et des denrées alimentaires ", Journal officiel de l'Union Européenne, C334, 31 oct. 2012, en ligne : http://eur-lex.europa.eu/legal-content/FR/TXT/?uri =uriserv:OJ.C_.2012.334.01.0011.01.FRA).

11. La survivance, voire l'affirmation, de deux options orthographiques divergentes cristallisée par la discordance d'écriture des deux entrées de dictionnaire citées plus haut - vient froisser la surface de ce consensus. À côté de la tendance majoritaire, qui respecte la règle anglaise exigeant le doublement de la consonne lors de l'adjonction $\mathrm{du}$ suffixe $-\mathrm{Y}$ après une voyelle brève monographique, se maintient et se radicalise la tradition inverse, qui semble prendre le parti de s'écarter de la norme étrangère afin de se rapprocher des us orthographiques de la langue d'accueil (implicitement posée comme étant le breton). Ce remodelage trouve son expression paroxystique dans l'entrée « Jonig » du dictionnaire bilingue breton/français des éditions Skol Vreizh, qui dépouille résolument le mot de toute marque exogène en faisant tomber le graphème $<\mathrm{h}>$ en plus de l'un des deux $<\mathrm{n}>$ et en substituant au diminutif anglais - Y la transposition bretonne -IG, donnant ainsi naissance à une forme canonique qui me semble procéder davantage d'une volonté de normalisation que de l'observation d'un corpus attesté (cf. Francis FAVEREAU, Dictionnaire du Breton contemporain bilingue, Morlaix, Skol Vreizh, 5e éd., 2000 [1992], p. 359). 
JOHNNY se glisse la plupart du temps dans le discours universitaire sans y faire aspérité :

« Deux chansons en anglais recueillies à Roscoff [lors des enquêtes d'ethnomusicologie conduites en 1951-1953] rappellent d'ailleurs l'importance des migrations économiques liées à la vente d'oignons en Angleterre par les Johnnies ${ }^{12}$. »

Rares sont les instances d'emploi où il fait encore figure de corps étranger, singularisé par un signalement typographique systématique ${ }^{13}$. Son assimilation se lit d'autant plus clairement que les termes propres au sociolecte des marchands d'oignons léonards continuent généralement, par contraste, d'être encadrés de guillemets pour les acceptions particulières de mots français et soulignés d'italiques pour les emprunts de provenance anglaise ou bretonne :

« De juillet à janvier ou février, les Johnies, des vendeurs organisés en "compagnies" et dirigés par un master, un maître, traversent la Manche pour y proposer au porte-à-porte, à pied ou à vélo, leurs chapelets ${ }^{14}$.»

$\mathrm{Ne}$ suscitant habituellement d'autre intervention métalinguistique qu'une éventuelle expansion paraphrastique - plus ou moins développée selon le degré de didacticité de l'ouvrage concerné mais d'ordinaire limitée à une simple apposition définitionnelle ${ }^{15}-$, le mot JOHNNY semble à présent investi d'un tel caractère d'évidence que le seul type de glose qui

12. Éva Guillorel, Barzaz Bro-Léon, une expérience inédite de collecte en Bretagne, Rennes, PUR/CRBC, coll. «Patrimoine Oral de Bretagne », 2012, p. 135.

13. Dans l'article de Dany Guillou-Beuzit par exemple, s'il est mis en question par des italiques lors de sa première occurrence au sein du titre interrogatif (cf. supra), le mot JOHNNY réapparaît ensuite dès la première phrase du corps du texte sous une forme typographiquement neutre : «Aujourd'hui, si vous demandez à un Roscovite le nom du premier des Johnnies, il vous répondra : Henri Ollivier, comme l'affirme le père Médard. » (D. GuILlou-BeuZiT, art. cit., p. 475).

14. Christian Bougeard, Gens de Bretagne, 1880-1960, Paris, éditions du Chêne, 2009, p. 103, note 1. On se reportera, pour une mise en œuvre filée de cette pratique de discrimination typographique, à François CABIOC'H, «Johnnies : les onion-men du Léon ", Ar Men, n 72, déc. 1995, p. 30-43.

15. La séquence explicative peut venir s'insérer à la suite du mot dont l'auteur entend éclairer la signification, comme dans l'exemple précité, ou bien au contraire le précéder en ménageant alors un effet d'annonce : «[C]'est en 1828, en période de surproduction que commence à s'organiser la migration saisonnière des marchands d'oignons, les célèbres Johnies. » (Annick CLÉAC’H, « Oignons », Dictionnaire du patrimoine breton, op. cit., p. 703). 
en reformule d'aventure le sens se borne à une transposition littérale de langue à langue :

« Johnny, n. m., pl. Johnnies Marchands d'oignons de la région de Santec, Roscoff (Nord du Finistère), qui vendent leurs oignons en Grande-Bretagne, spécialement au pays de Galles (où ils sont surnommés Johnny wynion “ Jeannot des oignons ”, mais aussi en Écosse ${ }^{16}$. »

Pointant implicitement l'origine prénominale du désignateur JOHNNY et sa valeur diminutive, cette paraphrase interlinguale sous-entend parfois un lien sémantique entre surnomination hypocoristique (ou supposée telle) et attribution privilégiée (conjecturale) du nom à des enfants :

«Paotred an ognon [..., "l] es gars aux oignons", ce sont ces hommes de Roscoff et des environs qui, à partir de 1828, traversèrent la Manche pour aller vendre "les petits oignons de Roscoff", où ils étaient très prisés. Ces " hommes "-là n'étaient parfois que de très jeunes adolescents, et même des enfants, (Yves Corre, une des victimes du naufrage de la goélette Paquebot 5, le 14 août 1899, n'a que dix ans ${ }^{17}$ ). Aux membres de cette corporation originale, les Britanniques donnèrent le nom de Johnnies, les “petits Jean" 18 . »

Mais à ce jour, je n'ai relevé au cours de ma prospection qu'un unique commentaire - dont le cantonnement en note infrapaginale symbolise bien la marginalité - qui, en soulevant la question de la date de diffusion de la désignation en contexte breton, attire l'attention sur les lacunes de notre compréhension du vocable JOHNNY appliqué aux pérégrins du pays de Léon :

« Il serait intéressant de savoir à quelle date le terme de Johnny

16. J. LE DÛ, op. cit., p. 161.

17. Né à Roscoff le 18 octobre 1883, Yves Corre allait en réalité atteindre l'âge de 16 ans au moment de sa disparition en mer, survenue au petit matin du 13 août 1899 (cf. transcription du jugement rendu le 13 mars 1900 par le Tribunal civil de première instance de Lannion, Arch. mun. Roscoff, I E 3, 1900, acte $\left.n^{\circ} 34\right)$. L'exagération de la jeunesse de la victime trouve vraisemblablement sa source dans une tradition orale recueillie par le père Médard (cf. Tad Medar, Paotred an Ognon, St-Brieuc, Les Presses Bretonnes, 1973, p. 127) et participe d'une veine sentimentaliste dont se teintent volontiers les récits attachés à la geste des marchands d'oignons léonards.

18. Fañch Morvannou, «La Métamorphose du Père Médard », dans P. Galliou, D. Banks (dir.), Apples of Gold : mélanges en mémoire de Daniel Le Gall, Brest, UBO, 2000, p. 171. (Au sein d'un passage très marqué typographiquement, la mise en italiques du mot «Johnnies » semble avoir une portée essentiellement autonymique, qui s'efface lorsque le texte perd sa dimension métalinguistique : "Chaque Johnny avait reçu une lettre », «plusieurs générations de Johnnies », ibidem.) 
s'impose pour désigner les colporteurs d'oignons. Le surnom breton de Johnniget est employé dans un reportage paru dans Le Courrier $d u$ Finistère en 1929 (Korn ar Johnniget, nov. déc. 1929) ; les publications de langue française l'emploient plus tardivement, semble-t-il ${ }^{19}$. »

Isolée, cette invitation à l'investigation historico-lexicologique est jusqu'ici restée lettre morte, laissant le champ libre à la propagation d'un discours doxique solidifié par d'inlassables répétitions, dont la survenance la plus récente offre un condensé saisissant : « [C]omme ils [ces Bretons] s'appellent tous "Petit Jean", on les appelle des Johnnies ${ }^{20}$. »

Devant la tonitruance de l'assertion cratyliste qui envisage la surnomination comme le décalque transparent de traits inhérents aux surnommés ${ }^{21}$ et veut lire dans un acte dénominatif atemporel (présent gnomique) émanant d'une instance baptiseuse indifférenciée (« on ») la reconnaissance d'un prétendu particularisme ethnique breton, il peut sembler sage de se retrancher dans la position de repli adoptée par Pierre-Jakez Hélias en pareille circonstance : «Que signifie ce nom ? Ce n'est pas facile à dire, malgré les assurances des archivistes de l'écriture et de la parole. Mais qu'importe ${ }^{22}$ !»

19. D. Guillou-Beuzit, art. cit., p. 481, note 20 (le retour des italiques marque ici encore l'emploi autonymique du mot « Johnny »).

J'ai repéré au détour d'un ouvrage d'histoire maritime une proposition de datation, non étayée mais plausible : " "Les Johnnies", c'était le terme donné [aux marchands d'oignons de la région de Roscoff] par leurs amis d'outre-manche depuis les années 1870. » (Henri FERmin, Les Bateaux anglais de Saint-Malo et le naufrage du vapeur Hilda, Dinard, Danclau, 1989, p. 107).

20. Yann QuÉFFÉLEC, Dictionnaire amoureux de la Bretagne, Paris, Plon/Fayard, 2013, p. 465. Souvent succincte, l'explication peut néanmoins prendre une forme plus étoffée : «Les prénoms portés à l'époque s'inspirent généralement du Nouveau Testament ou de la notoriété plus ou moins grande des saints populaires. Yann (Jean en français, John dans la langue de Shakespeare) fait partie des plus courants. Ce sont les Anglais qui ont vite fait de trouver un surnom à tous ces Yann qui parcourent leurs routes : John, ou plus affectueusement, Johnnies, les petits Jean, qualifiés chez les Bretons bretonnants d'ar Johnniged. » (J.T., «Les Johnnies, ces hommes enrichis en Grande-Bretagne », dans « Les Idées reçues sur la Bretagne », Historia/Ouest-France, hors-série, oct.-nov. 2012, p. 91).

21. Sur le rôle du processus de nomination dans la construction des référents sociaux, voir Sonia BRANCA-RosofF, "Approche discursive de la nomination/dénomination ", dans L'Acte de nommer, Une dynamique entre langue et discours, Paris, Presses de la Sorbonne Nouvelle, 2007, p. 13-22.

22. Pierre-Jakez HÉliAS, à propos du mot «bigouden», dans Au Pays du Cheval d'Orgueil, Paris, Plon, 1980, p. 5. Pour une synthèse diachronique des représentations liées à ce vocable, voir P.-R. GiOT, «La Population bigoudène», dans Jean Sutter 
Mais qui ne dit mot consent. Au risque de briser un tabou, je me demanderai donc ce qui s'est véritablement joué dans l'assignation originelle de la dénomination JOHNNY aux marchands d'oignons léonards. Ou pour dire autrement : les marchands d'oignons léonards ont-ils jamais été appelés « Petit Jean » par leurs interlocuteurs premiers?

Afin d'examiner posément la validité de l'équation mécaniste somme toute bien commode JOHNNY = «Petit Jean », il m'a paru prudent de commencer par établir la place avérée du nom personnel JEAN parmi les prénoms effectivement portés par les marchands d'oignons léonards au moment de l'avènement de leur surnom collectif, dont j'ai repéré la première occurrence attestée dans un article publié à Cardiff en 1877.

Un sondage pratiqué sur un échantillon de 480 migrants saisonniers nés entre 1847 et $1877^{23}$ montre que si le patronage spirituel le plus communément recherché était celui de la Vierge, le prénom de dévotion JEAN jouissait d'une faveur incontestable auprès des familles dans lesquelles se recrutaient les marchands d'oignons.

et al., La Luxation congénitale de la hanche, Paris, PUF/Institut National d'Études Démographiques, cahier $n^{\circ} 62,1972$, p. 13-17.

23. Mon relevé prend appui sur un recensement réalisé par Jean-Marie Balanant à partir des fiches matricules militaires des classes 1867-1897 récemment mises en ligne sur le site des Archives départementales du Finistère (URL : http://mnesys-portail. archives-finistere.fr/?id=recherche militaire, consulté le 24/07/2014). Se fondant sur les indications portées au sein de chaque feuillet individuel dans la rubrique " Localités successives habitées par suite de changement de domicile ou de résidence », J.-M. Balanant a pu établir une liste de près de 500 Finistériens ayant effectué au moins un séjour de plusieurs mois en Grande-Bretagne pendant la période de l'année correspondant traditionnellement à la «saison d'oignons", en alternance avec une résidence régulière dans la région de Roscoff. Le dépouillement de cet inventaire que j'ai légèrement amendé et ramené à 480 noms -, m'a permis de dresser une carte des communes de naissance de ces Finistériens migrateurs, confirmant le rôle moteur joué par Roscoff (dont faisait alors partie Santec) mais aussi l'étendue de l'aire de recrutement des vendeurs : Roscoff (253 hommes, soit 52,7\% de l'ensemble), St-Polde-Léon (72 h., 15\%), Plougoulm (43 h., 9\%), Sibiril (36 h., 7,5\%), Cléder (24 h., $5 \%$ ), Plouénan (14 h., 2,9\%), Plouescat (6 h., 1,3\%), Tréflaouénan (6 h., 1,3\%), La Feuillée (5 h., 1\%), Mespaul (5 h., 1\%), Plouzévédé (2 h., 0,4\%), Île de Batz (2 h., 0,4\%), St-Vougay (2 h., 0,4\%), 1 seul homme pour Brest, Carantec, Guiclan, Morlaix, Nantes, Paris, Plougar, Plounéour-Trez, Plounevez Lochrist et Tréflez. (Ces résultats demandent à être affinés, puisque la commune de naissance n'est pas toujours identique à la commune de résidence au moment de l'embauche.) 
Tabl. 1 : Composants prénominaux les plus répandus chez les marchands d'oignons léonards (éch. de 480 hommes nés entre 1847 et 1877)

\begin{tabular}{|c|c|c|c|}
\hline Rang & Composant prénominal & $\begin{array}{c}\text { Nombre } \\
\text { d'occurrences }\end{array}$ & $\begin{array}{c}\text { Part sur l'ensemble } \\
\text { des prénoms des MOL }\end{array}$ \\
\hline 1 & MARIE & 124 & $25,8 \%$ \\
\hline 2 & JEAN & 102 & $21,3 \%$ \\
\hline 3 & FRANÇOIS & 83 & $17,3 \%$ \\
\hline 4 & YVES & 46 & $9,6 \%$ \\
\hline 5 & JOSEPH & 26 & $5,4 \%$ \\
\hline 6 & PIERRE & 27 & $5,6 \%$ \\
\hline 7 & PAUL & 26 & $5,4 \%$ \\
\hline 8 & LOUIS & 25 & $5,2 \%$ \\
\hline 9 & HENRI & 22 & $4,6 \%$ \\
\hline 10 & OLIVIER & 22 & $4,6 \%$ \\
\hline
\end{tabular}

Le palmarès des dix éléments prénominaux les plus représentés (tabl. 1), au sein desquels JEAN occupe le deuxième rang, s'accorde ainsi avec les pratiques nominatives ayant cours à la même époque dans l'ensemble de la Bretagne, qui elles-mêmes, mis à part la popularité régionale de l'hagionyme $\mathrm{YVES}^{24}$, ne se distinguent guère des coutumes alors installées dans d'autres campagnes françaises ${ }^{25}$, où « un stock de prénoms réduits [sic], empruntés aux plus huppés des saints du calendrier chrétien ${ }^{26}$ » entraîne la « répétition des mêmes prénoms [...] familiaux précieusement thésaurisés ${ }^{27}{ }^{\prime}$ d'une génération à l'autre.

24. Sur l'émergence et la fortune du prénom Yves en Bretagne, voir Pierre-Yves QuÉMÉNER, «Le Prénom Yves, histoire d'un prénom breton », Le Lien du Centre Généalogique du Finistère, ${ }^{\circ} 124$, déc. 2012, p. 17-21 et n ${ }^{\circ} 125$, mars 2013, p. 45-52.

25. « [M]ême [... d]ans les lieux les plus imprégnés par la langue bretonne, les prénoms "bretons" de loin les plus courants sont Jean et Marie [...] . Cette situation se confirme jusqu'au XXe siècle : un échantillon de 6000 enfants nés au XIXe siècle livre ainsi $22 \%$ de Jean, $37 \%$ de Marie [...] et seule la présence d'Yves au sixième rang [...] marque une certaine originalité. [... U]n certain conformisme social a [...] dû peu à peu accentuer la marginalisation des prénoms propres à la Bretagne, d'autant que l'Église, au 19e surtout, promeut très fortement le culte marial : $45 \%$ des Bretons portent alors Marie pour second prénom. » A. CROIX, Dictionnaire du patrimoine breton, op. cit., p. 803.

26. Gérard VALLET, «Pratiques du baptême en Forez d'après les livres de raison (XVIe_ XVIII ${ }^{\mathrm{e}}$ siècles) », dans Guido Alfani, Philippe Castagnetti et Vincent Gourdon (dir.), Baptiser, pratique sacramentelle, pratique sociale (XVIe-XXe siècles), Saint-Étienne, Publications de l'Université de Saint-Étienne, 2009, p. 192.

27. Jérôme-Luther VIRET, «Un Hommage rendu à la parenté charnelle. Les prénoms multiples en Basse-Normandie au XVIII siècle », dans Baptiser..., op. cit., p. 223. 
Mais dans le Léon comme dans le reste de la France urbaine puis rurale, la nécessité pragmatique de différencier les individus, conjuguée au souci symbolique de renforcer les solidarités sociales locales en matérialisant la solidité des liens de parrainage/compérage par l'ouverture de la transmission des prénoms aux deux parents spirituels ainsi qu'aux parents naturels, conduisit à la prolifération des prénoms multiples ${ }^{28}$, qui constituent plus d'un tiers des prénoms recensés chez les marchands d'oignons ${ }^{29}$.

Trop commun pour pouvoir remplir à lui tout seul la fonction individuante attendue d'un nom propre ${ }^{30}$, JEAN fut touché au premier chef par cette vogue associative. Sur les 102 marchands d'oignons $(21,3 \%$ de l'ensemble) qui portent un prénom à composante johannique, seuls 16 vendeurs (soit 3,3\% du total) s'appellent simplement JEAN (tabl. 2).

Quid, alors, de l'affirmation selon laquelle le «surnom de "Johnny" [a été] donné à ces émigrants saisonniers par les Anglais, fort étonnés de rencontrer autant de Bretons prénommés Jean ${ }^{31} »$ ?

Le journaliste et écrivain gallois Gwyn Griffiths, qui depuis plus de trente ans œuvre avec verve à la vulgarisation de l'histoire des « Shonis », a suggéré que l'appellation collective JOHNNY proviendrait d'une déformation du prénom composé JEAN YVES :

"The only explanation I have ever heard for the nick-name "Johnny" on the onion-sellers is that so many of them had the Christian

28. Selon l'abbé Jean Feutren, « les doubles prénoms tels Jean-Marie » firent leur entrée dans les registres paroissiaux roscovites dès le milieu du XVII siècle (« Noms et surnoms : où l'on voit comment étaient tenus nos anciens registres », Bulletin paroissial de Roscoff, déc. 1974, n² 291, en ligne : http://www.roscoff-quotidien.eu/histoire-bulletinparoissial-291.htm). « [R]éponda[nt] avant tout au désir des parents de transmettre avec leur nom leurs prénoms ou ceux des divers ascendants[, 1]e procédé avait, en outre, l'avantage de réduire les risques d'homonymie. » («Appellations du passé », Bulletin paroissial de Roscoff, $\mathrm{n}^{\circ}$ 243, mai 1970, en ligne : http://www.roscoff-quotidien.eu/ histoire-bulletin-paroissial-243.htm).

29. Sur les 480 prénoms de marchands d'oignons pris en compte, 163 (soit $34 \%$ ) sont des composés, parmi lesquels dominent les prénoms doubles (150 occ., soit 31,3\%) bien que l'on trouve çà et là quelques prénoms triples (13 occ., soit $2,7 \%$ ), qui surviennent quand le parrain transmet son propre prénom double en bloc et/ou lorsque l'un des parents biologiques lègue également son prénom au baptisé.

30. «Ce qu'on entend ordinairement par nom propre est une marque conventionnelle d'identification sociale telle qu'elle puisse dégager constamment et de manière unique un individu unique ». Émile Benveniste, Problèmes de linguistique générale, t. 2, Paris, Gallimard, 1974, p. 200.

31. Yves Busson, « Bretagne : les Johnnies de Roscoff », Univers du Vivant, 1987, p. 39. 
name Jean-Yves. / La seule explication que j'aie jamais entendue à propos de l'attribution du surnom "Johnny" aux marchands d'oignons est que tant d'entre eux se prénommaient Jean-Yves ${ }^{32}$.»

"On les appelle les "Johnnies de Roscoff" ou encore, dans cette région où l'on parle beaucoup le breton, Ar Johniged. Leur surnom s'explique par le prénom de beaucoup d'entre eux, Jean ou Jean-Yves. Yves, du nom du saint patron préféré des Bretons, était un prénom très populaire en Bretagne ${ }^{33}$.»

Tabl. 2 : 10 prénoms simples les plus répandus chez les marchands d'oignons léonards (éch. de 480 hommes nés entre 1847 et 1877)

\begin{tabular}{|c|l|c|c|}
\hline Rang & Prénom simple & $\begin{array}{c}\text { Nombre } \\
\text { d'occurrences }\end{array}$ & $\begin{array}{c}\text { Part sur l'ensemble des } \\
\text { prénoms des MOL }\end{array}$ \\
\hline 1 & FRANÇOIS & 52 & $10,8 \%$ \\
\hline 2 & YVES & 25 & $5,2 \%$ \\
\hline 3 & PAUL & 20 & $4,2 \%$ \\
\hline 4 & OLIVIER & 19 & $4,0 \%$ \\
\hline 5 & JOSEPH & 18 & $3,8 \%$ \\
\hline 6 & HENRI & 17 & $3,5 \%$ \\
\hline 7 & JEAN & 16 & $3,3 \%$ \\
\hline 8 & JACQUES & 14 & $2,9 \%$ \\
\hline 9 & PIERRE & 14 & $2,9 \%$ \\
\hline 10 & ALAIN & 13 & $2,7 \%$ \\
\hline & Poids cumulé & 208 & $43,3 \%$ \\
\hline
\end{tabular}

Entrant dans la composition de 46 prénoms (soit 5,1\% de l'ensemble), l'élément YVES se place en quatrième position dans la base de données que j'ai étudiée. Apparaissant le plus souvent sous forme simple (25 occ.), il s'allie à MARIE en 17 occasions, au regard desquelles l'hapax «Jean Yves $^{34} \gg$ (tabl. 3) fait bien pâle figure, rendant peu vraisemblable, aussi

32. Gwyn GRIFFITHS, Goodbye, Johnny Onions, Redruth, Dyllansow Truran, 1987, p. 72. Cette explication est absente de l'édition originale galloise parue six ans plus tôt ( $Y$ Shonis Olaf, Llandysul, Gwasg Gomer, 1981, p. 92).

33. G. Griffiths, Le Monde des Johnnies, trad. Jocelyne Mondot, Brest, éditions Le Télégramme, 2002, p. 11. (La locution « Johnnies de Roscoff» est citée en français dans le texte anglais, The Last of the Onion Men, Llanrwst, Gwasg Carreg Gwalsh, 2002, p. 11.)

34. Jean Yves Corre, né à Cléder en 1874, séjourne à Portsmouth de juillet 1899 à mars 1900, puis à « Cap Martin, Angleterre » (Carmarthen ?) de juillet 1901 à février 1902 (Arch. dép. Finistère, 1 R 887-1894, n 734). Entre 1902 et 1905, lors des naissances 
engageante soit-elle, l'explication paronymique fondée sur la proximité phonique du nom JOHNNY (/dzDni/ en anglais, / §o:nI/ en gallois) avec une hypothétique réalisation hybride *John Yves ou *Sion Yves (/dzpni:v/ ou / Jo:ni:v/).

Tabl. 3 : part des prénoms à composante johannique chez les marchands d'oignons léonards (éch. de 480 hommes nés entre 1847 et 1877)

\begin{tabular}{|c|c|c|c|}
\hline & \multirow{3}{*}{$\begin{array}{c}\text { Nombre } \\
\text { d'occ. }\end{array}$} & \multirow{3}{*}{$\begin{array}{c}\begin{array}{c}\text { Part sur l'ensemble des } \\
\text { prénoms portés par les MOL }\end{array} \\
3,3 \% \\
\end{array}$} \\
\hline & & & \\
\hline Prénom simple & JEAN & & \\
\hline \multirow{9}{*}{$\begin{array}{l}\text { Prénoms doubles : } \\
\text { JEAN en position initiale }\end{array}$} & JEAN MARIE & 52 & $10,8 \%$ \\
\hline & JEAN FRANÇOIS & 8 & $1,7 \%$ \\
\hline & JEAN CLAUDE & 7 & $1,5 \%$ \\
\hline & JEAN PIERRE & 4 & $0,8 \%$ \\
\hline & JEAN BAPTISTE & 2 & $0,4 \%$ \\
\hline & JEAN LOUIS & 2 & $0,4 \%$ \\
\hline & JEAN PAUL & 2 & $0,4 \%$ \\
\hline & JEAN HIPPOLYTE & 1 & $0,2 \%$ \\
\hline & JEAN YVES & 1 & $0,2 \%$ \\
\hline $\begin{array}{l}\text { Prénom double : JEAN en } \\
2^{\mathrm{e}} \text { position }\end{array}$ & LOUIS JEAN & 1 & $0,2 \%$ \\
\hline \multicolumn{2}{|c|}{ Total des prénoms johanniques doubles } & 80 & $16,7 \%$ \\
\hline \multirow{5}{*}{$\begin{array}{l}\text { Prénoms triples : } \\
\text { JEAN (MARIE) en } \\
\text { position initiale }\end{array}$} & JEAN FRANÇOIS MARIE & 1 & $0,2 \%$ \\
\hline & JEAN MARIE PASCAL & 1 & $0,2 \%$ \\
\hline & \begin{tabular}{|l|} 
JEAN MARIE \\
TOUSSAINT
\end{tabular} & 1 & $0,2 \%$ \\
\hline & JEAN MARIE VICTOR & 1 & $0,2 \%$ \\
\hline & JEAN MARIE VIRGINIE & 1 & $0,2 \%$ \\
\hline $\begin{array}{l}\text { Prénom triple : JEAN en } \\
\text { position centrale } \\
\text { (JEAN MARIE en position } \\
\text { seconde) }\end{array}$ & JOSEPH JEAN MARIE & 1 & $0,2 \%$ \\
\hline \multicolumn{2}{|c|}{ Total des prénoms johanniques triples } & 6 & $1,3 \%$ \\
\hline \multicolumn{2}{|c|}{$\begin{array}{l}\text { Poids cumulé : ensemble des prénoms } \\
\text { à composante johannique }\end{array}$} & 102 & $21,3 \%$ \\
\hline
\end{tabular}

de ses enfants, il est inscrit à l'état civil sous la profession de " tailleur de pierres » ou « fendeur de rochers ». Employé dans les carrières de granit de Cléder, comme ses compatriotes Tanguy L'Aot (qui sera l'un des cinq Bretons rescapés du naufrage du Hilda de novembre 1905) et Hervé Le Bihan (disparu dans la catastrophe), il fait partie du petit groupe de marchands d'oignons dont le métier principal n'est pas le travail de la terre. (Je remercie Michèle Segura-Coz, qui m'a transmis ces deux noms.) 
Sans surprise, le prénom johannique le plus fréquemment attribué est JEAN MARIE, qui, réunissant les prénoms masculin et féminin les plus révérés, à l'image des statues de Saint Jean et de la Vierge appariées sur les poutres de gloire des églises bretonnes, nomme un peu plus d'un marchand d'oignons sur dix (52 occ. ; 10,8\%), à hauteur égale du prénom simple FRANÇOIS.

Bien que l'exiguïté de l'échantillon n'autorise qu'une comparaison circonspecte, l'analyse des données fournies par une centaine de passeports datant de 1857-1858 ${ }^{35}$ permet peut-être l'ébauche d'une mise en perspective diachronique (tabl. 4), qui semble suggérer que le milieu de recrutement des marchands d'oignons léonards aurait connu dans la seconde moitié du XIX ${ }^{e}$ siècle un relatif élargissement de son répertoire de prénoms masculins ${ }^{36}$, corrélé à une diminution proportionnelle de la place des prénoms johanniques, au moment même où le surnom JOHNNY allait surgir dans les sources écrites de langue anglaise et galloise.

Tabl. 4 : évolution de la part des prénoms comportant les composants JEAN et/ou FRANÇOIS chez les marchands d'oignons léonards dans la seconde moitié du XIXe siècle

\begin{tabular}{|l|c|c|}
\hline \multicolumn{1}{|c|}{ Prénom } & Passeports de 1857-1858 & Matricules militaires de 1867-1897 \\
\hline JEAN simple & $8,9 \%$ & $3,3 \%$ \\
\hline JEAN MARIE & $19,6 \%$ & $10,8 \%$ \\
\hline JEAN FRANÇOIS & $1,8 \%$ & $1,7 \%$ \\
\hline JEAN FRANÇOIS MARIE & $0,0 \%$ & $0,2 \%$ \\
\hline JEAN + autre & $4,5 \%$ & $5,4 \%$ \\
\hline FRANÇOIS simple & $12,5 \%$ & $10,8 \%$ \\
\hline FRANÇOIS MARIE & $1,8 \%$ & $2,3 \%$ \\
\hline FRANÇOIS + autre & $3,6 \%$ & $2,3 \%$ \\
\hline Poids cumulé & $52,7 \%$ & $36,8 \%$ \\
\hline
\end{tabular}

En 1895, parut à Londres un roman sentimental qui marqua l'entrée en littérature de la figure du marchand d'oignons breton. Comparé à saint

35. Deux séries d'enregistrements de passeports délivrés en juillet-août 1857 et 1858 à des « cultivateurs » accompagnant des chargements d'oignons vers l'Angleterre et le pays de Galles attestent des premières vagues de départs concertés connues (Arch. dép. Finistère, 4 M 379, enregistrements, 1854-1926). Ce sont les seules listes nominatives officielles un tant soit peu complètes qui nous soient parvenues pour la période 18501916.

36. On dénombre par exemple dans l'échantillon de 1847-1877 un Adolphe, un Victor, quatre Eugène et un rutilant Pascal Casimir, qui font sans doute écho aux modes françaises du temps. 
Jean pour son exotique beauté juvénile lors de sa rencontre fulgurante avec la narratrice-héroïne - que la jeune fermière anglaise dépeint sur le mode de l'apparition -, le personnage est prénommé... Yvon :

"The wind was 'twas beaten' the drops vrom the chestnut leaves agen 'my veace, and my hair out o' curl was vlyen' in my eyes, when my thoughts [...] was stapp'd, all on a zudden, by zeein'zome one comen' alang the rhoad [...]. I ztared wi' all my eyes at 'un! But 'twarn't zo much his outlandish clothes as his beautiful dark veace an rhed lips I did look at. Zich a handsome veace as 'twas! I'd never zeen a han'zom'r. His hair 'twas black as black, an' her laid wi' a bit of a curl in th' end of it rhight auv'r his two broad zhould'rs. Somehow, that black hair, her did meakes his white veace the whiter, the paleness of which show'd droo the zunburn. I hadn't never zeen a man wi' lang hair avore like a maid's! Leastways, not unless 'twere St Jan in mother's gurt Bible as laid to best parlour. Him had hair like thik ztraanger! An'as I minded it, I think'd, 'twarn't no wonder the Lord lov'd St Jan, if he did be like he. [...L]eanen' his two brown arms in the knitted jersey atop o' our low stone wall, the Breton straanger zays to I, "Santez An-na! Annaik! Mees An-na, - my name - Yvon - Yvon Dréano. I come from Roscoff, Finistère." / Le vent projetait les gouttes tombant des feuilles de marronnier contre mon visage, et faisait voler dans mes yeux les mèches folles de mes cheveux, lorsque mes pensées [...] furent interrompues, tout d'un coup, en voyant quelqu'un venir le long de la route [...]. Je le contemplais de tous mes yeux! Mais ce n'était pas tant ses étranges habits que son beau visage sombre et ses lèvres rouges que je regardais. Un si beau visage ! Je n'en avais jamais vu de plus beau! Ses cheveux étaient plus noirs que noirs, et retombaient en légères boucles sur ses deux larges épaules. Curieusement, la noirceur de ses cheveux faisait ressortir la blancheur de sa peau, dont la pâleur transparaissait à travers la brûlure du soleil. Je n'avais jamais vu d'homme aux cheveux longs comme ceux d'une fille! Ou bien alors St Jean dans la grande Bible que Mère gardait dans la pièce d'apparat. Ses cheveux étaient pareils à ceux de cet étranger ! Et, en y réfléchissant, je me disais qu'il n'était pas étonnant que le Seigneur eût aimé St Jean, s'il lui ressemblait. [...] Appuyant ses deux bras bruns dans leurs manches de tricot sur notre mur de pierre, l'étranger breton me dit : "Santez An-na! Annaik!

37. Dorénavant, je signalerai en les soulignant d'un trait les segments de texte que j'ai pris le parti de laisser en l'état, sans les traduire. Il s'agira soit de respecter la graphie des scripteurs sans multiplier les mentions « sic », soit de conserver leur hybridité aux représentations du parler des marchands d'oignons léonards telles qu'elles apparaissent dans les textes, ou encore, dans le cas de syntagmes désignatifs dominés par les noms JOHN(NY)/JACK, de ne pas fausser la démonstration par une traduction impliquant inévitablement un choix interprétatif. 
Mees An-na, - my name - Yvon - Yvon Dréano. I come from Roscoff, Finistère. $" 38$. "

$*$

Jusqu'au tournant du siècle, les principales sources britanniques faisant figurer des noms de marchands d'oignons sont les rarissimes actes administratifs qui nous sont parvenus et les nombreux comptes rendus d'audience de la presse régionale rapportant les divers incidents dans lesquels des Léonards se trouvaient impliqués, en tant que plaignants ou comme prévenus.

Les archives nationales de Londres conservent dans les registres maritimes douze pages sur lesquelles les officiers des douanes de Plymouth consignèrent, entre 1848 et 1858 , le débarquement de passagers étrangers venus de Roscoff ${ }^{39}$. L'examen de ces entrées nous livre, outre la plus ancienne occurrence attestée en anglais d'une désignation reconnaissant un Léonard comme marchand d'oignons ${ }^{40}$, un premier aperçu des diffé-

38. Alicia A. Leith, A Plant of lemon verbena : a Somerset idyll, Londres, Gibbings \& Company, 1895, p. 47-61.

À l'autre extrémité chronologique et géographique de l'histoire littéraire des marchands d'oignons léonards, le héros du roman naturaliste Johnny de Roscoff, dont la première évocation est également traversée par une imagerie solaire et religieuse à forte charge érotique, ne s'appelle pas Jean non plus : «-C'est Ribler, dit Jacques Castel, je reconnais son trot. Il avait le visage en pleine lumière et ses yeux, très bleus, s'alanguissaient encore dans le halo qui le nimbait. Sa chevelure en désordre se pailletait d'or. Dégagé, le front offrait sa sérénité. » (Yves-Marie RudEL, Johnny de Roscoff : roman, Paris, Librairie Celtique, 1945, phrase d'ouverture, p. 7).

39. The National Archives, Public Record Office, HO 3, Aliens Arrivals, pièces 50, 53, 54, 62, 66 et 94. Pour le XIXe siècle, ces douze pages sont apparemment les seules traces officielles de l'arrivée de navires en provenance de Roscoff dans les ports britanniques à ne pas avoir sombré dans un immense naufrage archivistique.

Après recoupement avec les registres d'état civil et les passeports pour l'intérieur et l'extérieur détenus par les archives municipales de Roscoff, les 47 noms relevés semblent pouvoir être mis en correspondance avec 28 voyageurs, que j'ai identifiés comme étant Esprit Dirou, Eugène Tanguy, François Quéau, François Quéméner, François Simon, Francois Tanguy, Gabriel Corre, Gabriel Roignant, Guillaume Foustel, Guillaume Postec, Guillaume Roignant, Hamon Créach, Hamon Quéméner, Henry Mouez, Hippolyte Quéméner, Jacques Tanguy, Jean Chapalain, Jean (Yves) Danielou, Jean Marie Créach, Jean Marie Kerleguer, Jean Marie Roignant, Jean Simon, Joseph Corre, Joseph Prigent, Joseph Quéméner, Joseph Tanguy, Olivier Creignou et Yves Guivarch.

40. Une entrée du 17 septembre 1858 signale le débarquement de « François Queon, Onion Merchant», accompagné de "François Simon», «Jean Simon» et «Kerlegueurs J Marie », tous trois «Labourer[s]» [calque lexical du français «laboureur »]. Né à Roscoff en 1824, François Quéau est enregistré comme «cultivateur» lors de sa 
rents traitements subis par les noms des immigrés bretons une fois la mer franchie.

Hormis quelques transcriptions fidèles à l'état civil français ${ }^{41}$, les inscriptions présentent des altérations morphographiques plus ou moins prononcées, dont certaines semblent être imputables à une lecture maladroite de papiers d'identité ${ }^{42}$, tandis que d'autres paraissent provenir plutôt d'une discrimination auditive approximative et/ou d'une hyperfrancisation des noms bretons ${ }^{43}$.

On remarque aussi l'anglicisation de 9 des 47 prénoms, dont 7 JEAN changés en «John », mais il s'avère délicat d'attribuer la responsabilité de cette adaptation. Est-elle à mettre au compte de l'officier des douanes ou à celui du capitaine du bateau ${ }^{44}$ ? Ou bien provient-elle de l'initiative de patrons, aguerris par les voyages commerciaux réguliers qui depuis plusieurs décennies conduisaient certaines familles léonardes bien audelà des confins de la Bretagne ${ }^{45}$ ? Comment interpréter, au sein d'une

demande de passeport pour Plymouth du 12 juillet 1858 (Arch. dép. Finistère, 4 M 379) et listé comme chef de compagnie («Fois Quéau et $\left.\mathrm{C}^{\mathrm{ie}} »\right)$ ) lors de la première des trois expéditions d'oignons qu'il effectue vers la Grande-Bretagne durant l'été 1858 (Service Historique de la Marine de Brest, Registre des entrées et sorties de bâtiments : quartier de Roscoff (1858-1878), 6P 94, entrée du 11 juil. 1858).

41. E.g. " Gabriel Corre », « Henry Mouez », « Henri Prigent ».

42. E.g. « Olivia Cragnon », "Yne Guivarch », « Henri Morez», « Roginant Gabriel », « J.M. Boignant».

43. E.g. « Joseph Quimner », « Joseph Lescour », « Esprit Dirout», « Jacque Tenguit ».

44. Conformément à la loi sur l'enregistrement des étrangers (« Act for the Registration of Aliens ») du 19 mai 1836, "The Master of every Vessel which shall arrive from Foreign Parts shall immediately on his arrival declare in writing to the Chief Officer of the Customs whether there is to the best of his knowledge any Alien on board of his Vessel [...] ; and shall in the said Declaration specify the number of Aliens (if any) on board his Vessel [...], and their Names, Rank, Occupation, Description, as far as he shall be informed thereof [.] / Le maitre de chaque vaisseau qui arrivera de provenances étrangères devra, immédiatement à son arrivée, faire savoir par écrit au principal officier des douanes s'il se trouve à sa connaissance quelque étranger à bord de son vaisseau [...]; et devra dans ladite déclaration, le cas échéant, spécifier le nombre d'étrangers se trouvant à bord de son vaisseau [...], et leurs noms, rang, profession, signalement, dans la mesure où ces informations auront été portées à sa connaissance [.] »

Les feuillets reliés conservés à Londres portant la mention «true copy» [copie authentique] et présentant pour certains une même écriture alors que les noms des capitaines cités sont différents, je pense qu'ils ont été remplis par un scripteur anglographe.

45. Les noms rencontrés dans les registres maritimes se retrouvent parmi ceux que distingue en 1845 le fondateur de la Société Royale d'Horticulture : «Il y a vingt ans, les jardiniers de Roscoff allaient eux-mêmes à Morlaix, et quelquefois à Brest, avec une charge, et quelquefois, mais rarement, deux charges de cheval ; maintenant chacun a une ou 
même entrée, la juxtaposition hétéroclite d'un «Guillaume » et d'un «William ${ }^{46}$ ? Que penser de redécoupages morphosémantiques hybrides tels que « John MarieCréach » / «John Mariecreach » ${ }^{47}$ ?

Les relations de procès publiées dans la presse provinciale à partir de 1865 ne permettent pas de répondre à ces interrogations, mais confirment et affirment les traits qui se dessinent dans les registres maritimes. Soumis à une anglicisation forcenée ${ }^{48}$ ("Charlie Ben » ${ }^{49}$, "Francis Kirbey ${ }^{50}$ ", « Frances Koth ${ }^{51} »$, « Francis Shapland ${ }^{52} »$, « Henry Gostill ${ }^{53} »$, « James Ruel $^{54} »$, «John French and John Cuff $^{55}$ », «Nicholas Gleddie ${ }^{56} »$, "Peter Vlock ${ }^{57} »$, «William Quex $\left.{ }^{58} »\right)$, accidentellement féminisés ( "Eugenie Roignant ${ }^{59} »$, «Mons. Francoise Le Grierch ${ }^{60} »$ ), écorchés jusqu'à en être défigurés (« Clode Pringent ${ }^{61}$ », « Guinard Casimarie ${ }^{62}$ »,

plusieurs voitures : aussi, les Roscovites ne se bornent plus à Morlaix ou à Brest ; ils vont, ils envoient au loin; ils expédient ou portent eux-mêmes leurs légumes à Nantes, à Angers, à Rennes, à Saint-Malo, à Paris, etc., etc. ; enfin, en Angleterre et jusqu'en Russie. [...] Les principaux jardiniers de Roscoff, ceux qui cultivent le mieux et sont considérés comme les plus habiles, sont MM. Olivier Séité, François-Marie Tanguy, les frères Allain, Joseph Craignon, Henri Olivier, les Daniélon, les Créack, Olivier Péron, François Jacob, Yves Quémène, Postec, Quéo, Jean Chapalain, etc. » (LouisÉtienne HÉRICART DE THURY, «État des cultures maraîchères à Roscoff », dans Journal d'Agriculture pratique et de jardinage, 2e série, t. 3, 1846, p. 207).

46. Entrée du 30 août 1852 : « Guillaume Foustel / William Postil / Merchants 》.

47. Entrées respectivement datées du 23 sept. et 13 déc. 1848.

48. Sur la pratique de traduction/adaptation du breton vers l'anglais chez les Victoriens comme « entreprise de domestication du peuple, du sauvage, de l'Autre », voir JeanYves LE DisEz, Étrange Bretagne, Récits de voyageurs britanniques en Bretagne (18301900), Rennes, PUR, 2002, p. 359-364.

49. The Aberystwyth Observer, 5 déc. 1885, p. 8.

50. The Western Daily Press, 21 août 1866, p. 3.

51. The Herald of Wales, 9 oct. 1897, p. 5.

52. The Western Daily Press, 3 nov. 1866, p. 3.

53. The Cheshire Observer, 20 oct. 1894, p. 8.

54. The Isle of Wight Observer, 12 sept. 1885, p. 5.

55. The Barry Dock News, 30 août 1900, p. 2. On devine un Jean Cueff derrière « John Cuff » (« cuff » signifie « manchette » ou « gifle » en anglais). Son compagnon « John French » était-il un Jean François X dont le second prénom aurait été pris pour le nom de famille, comme cela arriva vraisemblablement à «John Emile» quelques années plus tôt? (cf. The Hastings and St-Leonards Observer, 17 nov. 1894, p. 2.)

56. The Western Mail, 21 nov. 1895, p. 7.

57. The Cardiff Times, 12 déc. 1903, p. 5.

58. The Western Times, 10 sept. 1903, p. 5

59. The Cheshire Observer, 21 sept. 1889, p. 6.

60. The Sunderland Daily Echo, 28 sept. 1891, p. 3.

61. The Exeter and Plymouth Gazette, 30 sept. 1903, p. 5.

62. The Haverfordwest and Milford Telegraph, 20 sept. 1899, p. 3. 
« Julan Gueman ${ }^{63} »$, « Karlequer Frongis ${ }^{64} »$, « Larodd Teaf ${ }^{65} »$, «Pier Leagoff ${ }^{66} »$, «Salien Harmon $\left.{ }^{67} »\right)$ - non sans parfois quelque poésie ou humour fortuits ( $"$ Yris Meikel $^{68} »$, « Joseph Derrier and Carl Caraf $\left.{ }^{69} »\right)$-, les anthroponymes léonards sont couramment réduits à de méconnaissables hiéroglyphes dans ces articles destinés à un lectorat populaire, qui correspondait à la section de la population amenée à entrer en contact direct avec les marchands d'oignons.

Comme dans les registres maritimes, le nom d'un même homme pouvait donner lieu à des lectures discordantes, tel celui d'Yves Danielou ${ }^{70}$, réfracté alternativement en «Evan Daniel ${ }^{71}$ » et « Ynis Danellion ${ }^{72}$ » suite à l'« agression sauvage » dont le migrant fut victime à Cardiff en $1882^{73}$.

Ces réencodages multiples occultaient parfois l'identité d'origine des Bretons en cherchant ostensiblement à la restituer. Ainsi la transposition scrupuleuse de «James Daniels » en «Jacques Daniels », appliquant à la lettre les conventions d'équivalence entre prénoms anglais et français, rendait-elle encore plus opaque le masque onomastique derrière lequel était dissimulé Jean Marie Danielou ${ }^{74}$, que ses compatriotes bretonnants appelaient entre eux « Jamar Catel » :

63. The Sunderland Daily Echo, 11 oct. 1902, p. 4.

64. The Weekly Mail, 11 sept. 1897, p. 10.

65. The Portsmouth Evening News, 18 sept. 1899, p. 3.

66. The Cambrian News, 18 août 1898, p. 6.

67. The Royall Cornwall Gazette, 29 sept. 1898, p. 6.

68. The South Wales Daily Post, 24 janv. 1898, p. 3.

69. The Exeter Flying Post, 12 août 1893, p. 7.

70. Né d'un père «cultivateur [...] actuellement absent de cette commune » (Arch. mun. Roscoff, I E 1, 1858, acte ${ }^{\circ} 56$ ), Yves Marie Danielou est déclaré «marchand des quatre saisons domicilié à Paris » à la naissance de son premier enfant (Arch. mun. Roscoff, I E1, 1887, acte $n^{\circ} 171$ ), puis « journalier [...] absent » lors de naissances ultérieures (e.g. Arch. mun. Roscoff, I E 1, 1889, acte $\mathrm{n}^{\circ} 101$ ). Sa fiche matricule (Arch. dép. Finistère, 1 R 886, cl. 1878, n 223) le situe à Paris en 1883, puis à Portsmouth en 1890.

71. « Savage assault on a French onion vendor», The Western Mail, 3 oct. 1882, p. 3.

72. «Assaults», The Bristol Mercury, 4 oct. 1882, p. 6.

73. Il est là encore malaisé de déterminer à quel endroit de la chaîne de communication a eu lieu le brouillage morphosémantique, mais on peut supposer qu' "Evan Daniel » émanerait d'une interprétation auditive alors qu' «Ynis Danellion» résulterait du déchiffrement d'un écrit.

74. Jean Marie Danielou, né à Roscoff en 1845 (Arch. mun. Roscoff, I E 1, 1845, acte n 66), est désigné comme « marchands de légumes » lors de la naissance de son premier enfant (Arch. mun. Roscoff, I E 1, 1869, acte $\mathrm{n}^{\circ}$ 93) et comme « cultivateur absent » lors de quatre naissances ultérieures $(1873,1875,1883$ et 1884). Lors du décès de son épouse, Marie Jeanne Ollivier - nièce d'Henri Ollivier, en qui la tradition roscovite voit le père fondateur des «Johnnies»-, il est dit «marchand de légumes, [...] actuellement en 
« James Daniels, a French onion seller living in Limelkiln-lane in a house by himself, was found dead on Sunday morning [.] / James Daniels, un marchand d'oignons français vivant seul dans une maison de Limekiln-lane, a été retrouvé sans vie dimanche $\operatorname{matin}^{75}[$.$] »$

«A French onion seller named Jacques Daniels, who had been resident in Portmadoc for about fifteen years, was found dead on Sunday morning in bed. He lived alone, and was found by the police, who burst the door open. / Un marchand d'oignons français nommé Jacques Daniels, qui résidait à Porthmadog depuis environ quinze ans, a été retrouvé sans vie dimanche matin dans son lit. Il vivait seul, et a été découvert par la police, qui a enfoncé la porte ${ }^{76}$. »

« Jamar Catel mort a Portmadoc au commencement de Janvier $1905 »(\text { illustr. 1) })^{77}$.

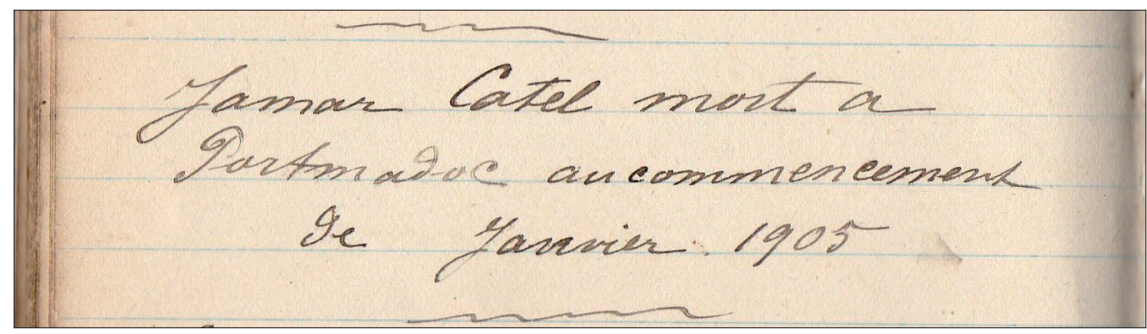

Illustr. 1 : extrait du carnet de Jean Marie Corre, p. 41 (coll. part.).

Aussi nombreux qu'aient été les marchands d'oignons à porter le nom de baptême JEAN MARIE, ce prénom foncièrement léonard parce que

Angleterre et absent de la commune depuis deux ans ». Je n'ai pour l'instant pas réussi à retrouver son matricule militaire ni la transcription de son acte de décès.

75. «Onion seller's sad end», The Cambrian News, 6 janv. 1905, p. 3.

76. «Found dead», The Welsh Coast Pioneer, 6 janv. 1905, p. 11.

77. J'ai pu remonter la piste de Jean Marie Danielou (surnommé « Catel » en référence au prénom de sa mère, Catherine Moncus) grâce à cette mention que j'ai découverte dans un carnet manuscrit conservé par Anne-Marie Kervellec, dont le grand-père, Jean-Marie Corre (1866-1949), avait été marchand d'oignons en Écosse de 1891 à 1900. Au fil des pages de ce(tte ?) « Mémoire », J.-M. Corre consigna en français, entre 1902 et 1914, les événements marquants qui rythmaient son quotidien : tâches agricoles, départs et retours de ses trois frères cadets de part et d'autre de la Manche, naissances, mariages et décès survenus dans son voisinage, mais aussi en août 1902, la résistance de la population roscovite à l'expulsion des Soeurs du St-Esprit. Je remercie très chaleureusement A.-M. Kervellec de m'avoir autorisée à prendre et donner connaissance de ce document unique, qui ouvre une fenêtre sur l'univers mental et linguistique d'un Roscovite situé au cœur du réseau des marchands d'oignons à une époque où leur activité battait son plein. 
quintessentiellement catholique se révèle avoir été tout particulièrement difficile à entendre, lire et reproduire pour leurs allocutaires britanniques. Tandis que la forme usuelle bretonnisée JAMAR(I ${ }^{78}$ se voyait adaptée en « James » ${ }^{79}$ ou transcrite tant bien que mal ( « Jamaris Rous ${ }^{80} »$, « Samarie Malgorn $\left.{ }^{81} »\right)$, la forme d'état civil n'était pas toujours décryptée comme une locution prénominale liée, l'étrangeté de l'élément marial faisant interférence, entravant le processus d'interprétation et bloquant toute velléité d'assimilation $^{82}$ :

«Charles Johnson, fisherman, Horncastle Road, was summoned for assaulting Creignon Jean Marie, hawker, on the $14^{\text {th }}$ inst. - Every year, a number of poor Frenchmen bring over cargoes of onions, which they carry about for sale strung on poles. In this case it was shown that defendant and some companions encountered Marie in Wide Bargate, when the defendant tripped him up, scattering his onions on the ground, and then assaulted him. / Charles Johnson, pêcheur, résidant à Horncastle Road, a comparu pour l'agression de Creignon Jean Marie, perpétrée le 14 de ce mois. Chaque année, un certain nombre de pauvres Français font venir chez nous des cargaisons d'oignons, qu'ils vendent en tresses portées sur des bâtons. Dans le cas présent, il s'avère que l'inculpé, qui se trouvait en compagnie d'autres hommes, a rencontré Marie dans Wide Bargate, où il lui a fait un croc-en-jambe, éparpillant ses oignons sur le sol, puis l'a agressé ${ }^{83}$. »

S'insinuant dans un entrefilet d'apparence par ailleurs factuelle, une brève parenthèse onomastique, se gaussant à demi-mots de l'association incongrue d'un nom hellénique et d'un colporteur étranger (ivrogne et baragouineur), témoigne de la condescendance - amusée, cinglante,

78. Sur les formes oralisées des prénoms léonards, voir Mikael MADEG, Anoiou-badiziant Bro-Leon, Brest, Emgleo Breiz, 2006, p. 18-26.

79. Un article écossais de 1923 présentait le patron Jean François Marie Tanguy (Roscoff, 1870 ) et son fils Jean Marie (Roscoff, 1900) respectivement comme «James Tanguy and his [...] son [...] James » («A Day with the "onion boys": picturesque Bretons, annual visit to Dundee », The Dundee Courier, 22 sept. 1923, p. 3). Soixante ans plus tard, Gwyn Griffiths rapportait que « in Roscoff Jean-Marie Cueff stopped being Jimmy and became Shamar or Sham-Bar. / à Roscoff, Jean-Marie Cueff cessait d'être Jimmy [- forme diminutive standard du prénom JAMES - ] pour devenir Shamar, ou ShamBar. » (G. GRIFFITHS, Goodbye, Johnny Onions, op. cit., p. 72).

80. The Portsmouth Evening News, 17 oct. 1883, p. 3.

81. The Haverfordwest and Milford Telegraph, 20 sept. 1899, p. 3.

82. Mon corpus ne contient aucun exemple de *John Mary.

83. «Boston police court», The Sheffield Daily Telegraph, 28 sept. 1904, p. 4. 
hargneuse ou compatissante - qu'exhale bien souvent le discours britannique portant sur les marchands d'oignons léonards :

«A French onion vendor, rejoicing in the somewhat classical name of Bernicot Heyppolite ${ }^{84}$, appeared before Bailie Peffers in Forfar Police Court yesterday, charged with having been drunk, brandishing a stick, and gathering a crowd around him in Castle Street the previous evening. Bernicot's knowledge of English appeared to be limited to the trade vocabulary connected with the disposal of onions, and, after evidence, he was called upon to pay $5 \mathrm{~s}$, or to pass other twenty-four hours in confinement. / Un vendeur d'oignons, enorgueilli du nom aux accents antiques de Bernicot Heyppolite, a comparu hier au tribunal de police de Forfar devant le juge Peffers, accusé d'avoir, la veille au soir, en état d'ivresse, brandi un bâton et provoqué un attroupement dans Castle Street. Sa connaissance de l'anglais paraissait restreinte au vocabulaire commercial lié à la vente d'oignons, et, après audition des témoins, il fut appelé à payer 5 shillings d'amende, ou à passer vingtquatre heures supplémentaires en détention ${ }^{85}$. »

Ces neuf mots entre virgules représentent aussi, sur le millier de textes que compte mon corpus britannique, l'un des deux seuls commentaires faisant montre d'une quelconque réaction face aux noms de ces hommes et enfants ${ }^{86}$.

Hors de l'enceinte des tribunaux, dans les rues et sur le seuil des maisons, dans cet espace liminal ${ }^{87}$ où se déroulait l'essentiel des

84. Une fiche matricule au nom d' « Hippolyte Bernicot» (Arch. dép. Finistère, 1 R 920, cl. $1880, \mathrm{n}^{\circ}$ 501) reporte, pour la période 1886-1901, des séjours annuels de 5 à 6 mois en Écosse. L'acte de naissance du futur marchand d'oignons indique que le père ne sait pas signer et entre le prénom HIPPOLYTE sous la graphie hypercorrecte « Hypolithe » (Arch. mun. Roscoff, I E 1, 1860, p. 3). Le Roscovite, alors déclaré comme « journalier », décédera à l'hospice de Quimper à l'âge de quarante-six ans (Arch. mun. Roscoff, I E 3, 1906 , acte $\left.\mathrm{n}^{\circ} 24\right)$.

85. «Local News », The Dundee Courier, 29 août 1903, p. 3.

86. Tout aussi laconique et sans appel, la seconde observation, parue à trois ans d'intervalle dans la même publication, présente une coquille qui semble transposer graphiquement le bredouillement auquel étaient couramment ravalés les noms bretons : «A young lad with an unpronouncable [sic] name, described as an onion seller, was brought into the Central Office in a drunken condition. He was one of the young lads who vend French onions in the autumn from door to door. / Un jeune garçon doté d'un nom imprononcable [sic], décrit comme vendeur d'oignons, a été conduit au tribunal en état d'ivresse. Il s'agit d'un des jeunes garçons qui vendent des oignons français au porte-àporte durant l'automne. » («Dundee Police Court», The Dundee Courier, 22 nov. 1906, p. 2).

87. Sur l'ambiguité de ces lieux stratégiques, à la fois points de contact et frontières, au sein de la géographie sociale victorienne, voir «House and Home » et «The Street », dans 
transactions sociolangagières auxquelles prenaient part les marchands d'oignons léonards pendant leur période d'exode, les noms personnels des immigrés semblent avoir été tout simplement hors sujet. Les documents que j'ai pu rassembler à ce jour ne permettent pas de penser que les vendeurs se seraient vus régulièrement demander leurs noms par leurs interlocuteurs quotidiens, ou qu'ils auraient été, pour beaucoup d'entre eux, en mesure de répondre adéquatement s'ils avaient reçu une telle marque d'attention:

"For about half a year, they are residents in our towns and cities, "strangers and foreigners", seldom hearing a kindly word. Their English is limited - "Want buy onions?" and "Wery sheap" being about all that some of them know [.] / Pendant près d'une demi-année, ils résident dans nos petites et grandes villes, "des étrangers et des gens du dehors ${ }^{88}$ ", entendant rarement un mot gentil. Leur anglais est limité - "Want buy onions ?" et "Wery sheap ${ }^{89}$ " étant à peu près tout ce que connaissent certains ${ }^{90}$. »

Installé au pays de Galles depuis une trentaine d'années, bien mieux

Judith Flanders, The Victorian House, Londres, Harper Perennial, 2004 [2003], p. xixlii et 349-379.

88. «Ainsi donc, vous n'êtes plus des étrangers, ni des gens du dehors ; mais vous êtes concitoyens des saints, gens de la maison de Dieu. » Épitre aux Éphésiens, 2-19.

89. Le troisième épisode des tribulations initiatiques du «mousse » Perig entre Roscoff et l'Écosse à l'été 1891 décrit avec humour l'apprentissage expéditif dispensé par les aînés pendant la traversée - les novices étant envoyés à l'aventure avec pour seul bagage linguistique la réclame "Onions, Mistress, very cheap » (Le Courrier du Finistère, «Korn ar Johnniget », 23 nov. 1929, p. 2). Avant d'être immortalisé en Bretagne par l'abbé Conq, le slogan de prédilection des marchands d'oignons léonards avait acquis un statut proverbial en Grande-Bretagne : «The subscription to Dunheved football club is only 2s.6d. That is like the French onions - "vara sheep" / La cotisation au club de football de Dunheved n'est que de deux shillings et six pennies. C'est comme les oignons français - "vara sheep". » («Football notes », The Royal Cornwall Gazette, 21 déc. 1893, p. 5).

90. M.-J.-K. CHAPMAN, « Work among onion boys », The Independent and Non-Conformist, 28 nov. 1890 , p. 187.

Parfois prise en compte comme circonstance atténuante en cas de délit, l'insécurité linguistique d'une grande partie des immigrés léonards - reflet de leur extrême vulnérabilité sociale - ne suscitait bien souvent que des réactions de commisération moqueuse : "A foreign onion dealer, who caused great amusement in giving his evidence, said that while near the Market on Saturday night defendant "fisted him and fought him", "broke all his clothes", and "stole his onions". / Un vendeur d'oignons étranger, qui provoqua beaucoup d'amusement en donnant sa déposition, raconta qu'alors qu'il se trouvait près du marché samedi soir, l'accusé le "coup-de-poigna et le combattit", "lui cassa tous ses habits" et "lui vola ses oignons". » (" Onion dealers in trouble », The Sunderland Daily Echo, 15 oct. 1878, p. 4). 
intégré socialement et linguistiquement que beaucoup de ses collègues, Jean Marie Danielou n'était néanmoins connu de ses clients et voisins gallois que par son cri de vente, qui lui tenait lieu de toute identité :

«[After he] came over to this country some thirty years ago, settling down first in Aberystwyth and moving to Portmadoc ten years later [... James Daniels] was a well-known character in the district, having learnt a good deal of Welsh and English. His other name being almost unknown, he was known by the phrase with which he introduced business when vending his wares, "Onions, Mary bach". The funeral took place on Tuesday, when scores of the juvenile acquaintances of "Onions, Mary bach" assembled outside the mortuary and accompanied the hearse down High-street. / [A]rrivé dans notre pays voilà près de trente ans, s'installant d'abord à Aberystwyth, avant de déménager à Porthmadog dix ans plus tard [... James Daniels] était un personnage bien connu du quartier, ayant acquis de bonnes connaissances en gallois et en anglais. Son autre nom étant presque inconnu, on le connaissait ${ }^{91}$ par l'expression qu'il utilisait pour entrer en affaire lorsqu'il vendait sa marchandise : "Onions, Mary bach". Lors de ses obsèques, mardi dernier, des dizaines de connaissances juvéniles d'“Onions, Mary bach" se sont rassemblées devant la morgue et ont accompagné le corbillard le long de la grand rue ${ }^{92}$. »

Frappé d'insignifiance, le nom de naissance était rabaissé au rang d' « autre nom/nom autre », cependant que l'individu s'effaçait derrière le «personnage ».

Dans l'Europe du XIX ${ }^{\mathrm{e}}$ siècle, où le discours public était contrôlé par des " bourgeoisies sédentaires luttant contre la concurrence des itinérants ${ }^{93}$ ", « [1] colporteur familier [...] et ses semblables [restaient en effet perçus comme] des étrangers, sans nom, tous identiques ${ }^{94} »$, auxquels ne pouvaient s'appliquer que des « appellation[s] niveleuse[s] collective[s] ${ }^{95}$ ». Et dans la "nation de boutiquiers » qu'était alors la Grande-Bretagne, « [b]y the middle of the nineteenth century, the social position of street

91. Je respecte volontairement dans ma traduction la triple variation sur le verbe KNOW («well-known», « unknown», « known»), qui me paraît mettre en évidence la contradiction fondamentale sur laquelle reposait la position des marchands d'oignons léonards en Grande-Bretagne, qui étaient, paradoxalement, d'autant plus « inconnus » qu'ils faisaient l'effet d'être « bien connus ».

92. The Cambrian News, 6 janv. 1905, p. 3.

93. Laurence FonTAINE, Histoire du colportage en Europe, XVe-XIXe siècle, Paris, Albin Michel, coll. L'Évolution de l'Humanité, 1993, p. 208.

94. Ibidem.

95. Ibid. 
sellers sank to its nadir, where it remained. [...] Hawkers [were] alter[ed] from living human beings to representations, stereotypes and cyphers. / à compter du milieu du dix-neuvième siècle, la position sociale des vendeurs de rue chuta à son nadir, pour ne plus s'en relever. [...] D'êtres humains vivants, les colporteurs furent réduits à des représentations, des stéréotypes et des figures ${ }^{96}$. »

Stigmatisés par l'exotisme spatio-temporel de leur vêture, les marchands d'oignons léonards étaient spécialement affectés par ce phénomène de dépersonnalisation/typification ${ }^{97}$ :

« A DUNDEE STREET FIGURE. He is an interesting study, the Brittany onion boy. Of course you know the kind I mean [.] There are about a score of them in Dundee at the present moment. [...] The Brittany onion boy is a picturesque figure as he wanders from house to house in his blue baker's cap, loose navy blouse [which] mark him off in a crowd as a typical representative of old-fashioned Brittany ${ }^{98}$. / UNE FIGURE DES RUES DE DunDEE. C'est un objet d'étude intéressant que le garçon-auxoignons de Bretagne. Vous voyez bien sûr de quelle sorte je veux parler [.] Il y en a environ une vingtaine à Dundee à l'heure actuelle. [...] Le garçon-aux-oignons est une figure pittoresque quand il erre de maison en maison avec son bonnet de boulanger bleu et son ample vareuse marine, [qui] le démarquent de la foule et le désignent comme représentant typique de l'archaïque Bretagne ${ }^{99}$.»

La «mise à l'écart par les mots ${ }^{100} »$ qui déniait à tous les vendeurs

96. Sean Sheshgreen, Images of the outcast, The Urban poor in the Cries of London, Manchester, Manchester University Press, p. 9-12.

97. Ce point de vue stéréotypant se traduit grammaticalement par la récurrence du schéma nominal THE $+\mathrm{N}_{\mathrm{SG}}$, e.g. : "Can the son of France not sell his onions today? » (Portsmouth, 1895), "But what of the onion-seller?» (Dundee, 1897), "The French onion vendor is in evidence at this time of the year » (Hastings, 1900), "The onion boy is an adept at " turning on the tap » when he confronts a customer » (Exeter, 1906), etc..

98. La parole uniformisatrice pouvait quitter le champ du folklorisme pour emprunter à celui d'un naturalisme darwinien plus brutalement déshumanisant : "bang! goes the knocker again. We answer the door. This time it is an unshaved specimen of Breton labouring life, who offers more onions for sale / Boum! Le heurtoir retentit de nouveau. Nous répondons à la porte. Ce coup-ci, c'est un spécimen mal rasé de la vie laborieuse bretonne, qui propose encore des oignons» («Perambulating Onion-men», The Guernsey Star, 27 nov. 1894, p. 3).

99. «Onions, 1d a pound», The Dundee Evening Telegraph, 8 nov. 1900, p. 3. À propos du « déni de contemporanéité » que manifeste la saturation du discours victorien sur les Bretons par la notion de costume, voir J.-Y. LE DiSEZ, op. cit., en particulier p. 340-343. 100. L. Fontaine, op. cit., p. 208. 
ambulants la dignité de sujet singulier ${ }^{101}$ touchait donc les nomades bretons avec une force décuplée. Si « [p]artout, le terme de colporteur se connot[ait] de nuances dépréciatives ${ }^{102}$ », en Grande-Bretagne, celui de « vendeur d'oignons » se chargeait parfois des fantasmes xénophobes et racistes les plus crus ${ }^{103}$ :

«[T]he horrid secret is out.... An onion-seller ... the very words stink in the nostrils and are fatal to romance. Fatal to romance in the minds of the fastidious, fatal to respectability in those of the common people, for only foreigners sold onions. Strange men with rings in their ears and long, dark curls like a woman's, and an eye that was at once bold and soft. / Voici révélé l'effroyable secret : c'était un vendeur d'oignons - les mots mêmes puent aux narines et sont fatals à l'amour. Fatals à l'amour dans l'esprit des personnes de qualité, fatals à la respectabilité aux yeux des gens du commun, car seuls les étrangers vendaient des oignons ${ }^{104}$. Des hommes étranges aux oreilles percées d'anneaux, aux

101. Sur la privation de "renommée » des individus socialement catalogués comme « infâmes », voir Giacomo TODESCHINI, Au pays des sans-nom : Gens de mauvaise vie, personnes suspectes ou ordinaires du Moyen Âge à l'époque moderne, trad. Nathalie Gailius, Lagrasse, éditions Verdier, 2005.

102. L. FONTAINE, op. cit., p. 208.

103. La violence chauvine prenant pour cible les marchands d'oignons léonards ne s'exprimait pas seulement dans les pages des romans : "Edwin Richards, a drover, was summoned by Jean Craignon for assaulting him outside the market on Thursday. Complainant, through an interpreter, said he was outside the market selling onions when the defendant and his son came up and capsized the cart. When he was picking up the onions defendant struck him violently, making his nose and mouth bleed. Charles Sellick said he was near the market, and when he saw defendant strike the young Frenchman he interfered, and defendant said, "Let the French go home to their own country." The brutish fellow, when he found he could not vent his rage any further on the poor little foreigner, set a sheep dog at him. / Edwin Richards, conducteur de bétail, a été cité à comparaître par Jean Craignon pour l'avoir agressé devant le marché jeudi dernier. Le plaignant, par l'intermédiaire d'un interprète, a déclaré qu'il se trouvait devant le marché à vendre des oignons lorsque l'accusé et son fils se sont approchés et ont renversé sa charrette. Tandis qu'il ramassait ses oignons, l'accusé l'a violemment frappé, le faisant saigner au nez et à la bouche. - Charles Sellick a témoigné qu'étant à proximité du marché, il est intervenu quand il a vu l'accusé frapper le jeune Français puis s'écrier : "Que les Français retournent dans leur propre pays!" Ne trouvant plus d'autre moyen de déverser sa rage sur le pauvre petit étranger, la brute lâcha sur lui son chien de berger. » (The Western Times, 12 sept. 1870, p. 2).

104. Attestée par la littérature et l'iconographie dès le début de la Renaissance, la vente d'oignons itinérante offrait encore au milieu du XIXe siècle un moyen de subsistance aux populations urbaines britanniques les plus démunies, chômeurs de longue durée, veuves sans ressources, et jeunes immigrées irlandaises déracinées dans les bas-fonds londoniens (cf. Henry MAYHEW, «Of onion-selling in the streets », London Labour and the London poor, Londres, Griffin, Bohn and Company, 1862, vol. I, p. 93-94). Les 
longues boucles noires pareilles à celles des femmes, et dont le regard était tout à la fois caressant et audacieux ${ }^{105}$. »”

Or, « [c]'est un phénomène aussi vieux que la langue : soustraits à leur vocation d'attributs de l'intimité, les prénoms s'utilisent à des fins de malveillance $^{106}$. » En anglais comme dans les autres langues européennes, le plus prisé des noms baptismaux ${ }^{107}$ s'était dégradé par effet d'usure sémantique jusqu'à devenir l'anti-prénom par excellence, vecteur de généricité et marqueur d'altérité, souvent teinté d'une vive coloration dépréciative :

«I know not how it has happened that in the principal modern languages John, or its equivalent, is a name of contempt, or at least of slight. So the Italians use Gianni, from whence Zani; the Spaniards Juan, as Bobo Juan, a foolish John; the French Jean, with various additions; and in English, when we call a man John, we do not mean it as a title of honour. / Je ne sais comment il est advenu que dans les principales langues modernes $\underline{\text { John }}$, ou son équivalent, soit un nom infamant, ou du moins offensant. Ainsi les Italiens font usage de Gianni, d'où nous tirons le mot Zani ${ }^{108}$; les Espagnols de Juan, dans l'expression Bobo Juan, John le benêt; les Français de Jean, auquel ils ajoutent divers qualificatifs; et en anglais, lorsque nous appelons un homme $\underline{\text { John, }}$, nous n'entendons pas lui conférer un titre honorifique ${ }^{109}$. »

«John. Heb. Gracious. Giovanni in Italian. Jean in French. The commonest Christian name in use, given originally from the most

saisonniers bretons ne firent ainsi que s'insérer dans un marché préexistant, adoptant puis adaptant les pratiques locales, avant de finir par supplanter ceux et celles qu'ils avaient d'abord imités et concurrencés. Bien qu'il arrive encore parfois de croiser dans les textes quelques colporteurs ou colporteuses indigènes jusqu'en 1895, toutes les mentions de marchands d'oignons ambulants rencontrées après 1900 concernent, à ma connaissance, les Léonards.

105. Fryniwyd TENNYSON JeSSE, The White riband, or a young female's folly, New York, George H. Doran Company, 1921, p. 32.

106. Maurice GILLET, Le Jean-foutre et la marie-salope, les prénoms dénigrés, dévoyés ou encanaillés du Moyen-Âge à nos jours, 2013, p. 1, en ligne : http://library.wobook.com/ WBuo2vf3eF3a-320/Le-jean-foutre-et-la-marie-salope.html.

107. Sur l'ancrage du prénom JOHN en Grande-Bretagne, voir George ReDMONDS, Christian names in local and family history, Toronto, Dundurn Press, 2004, notamment p. 3035 et $170-175$.

108. «Bouffon ». Cf. Thomas Whitcombe Greene, Old words and modern meanings, Londres, Longman and Company, 1876, p. 313.

109. Thomas TYRWhitT, The Canterbury Tales of Chaucer, vol. IV, Londres, William Pickering, 1830, p. 316 [note sur le vers 14816]. 
amiable of the Apostles. It's being so great a favourite seems at last to have turned the tables upon it, and brought it's [sic] familiarity into disrepute. / John. Hébreux. Plein de grâce. Giovanni en italien. Jean en français. Le plus commun des prénoms en usage, donné à l'origine en honneur du plus aimable des apôtres. Son immense popularité semble avoir fini par se retourner contre lui et porter discrédit à sa familiarité ${ }^{110}$. »

Le suffixe diminutif $-Y$ venant adjoindre le sème de la petitesse ${ }^{111}$, connoté négativement (peut-être par contamination homonymique avec la désinence adjectivale péjorative $-\mathrm{Y}^{112}$ ), à celui de l'indistinction/vulgarité que portait l'acception déclassée de JOHN, le dérivé JOHNNY faisait communément office de pantonyme familier «[a]pplied humorously or contemptuously to various classes of men [, synonymous with] fellow, chap. I appliqué avec humour ou mépris à diverses classes d'hommes [, synonyme de] "gars", ou "type" $113 »$ :

« Jeeves - my man, you know - is really a most extraordinary chap. So capable. Honestly, I shouldn't know what to do without him. On broader lines he's like those chappies who sit peering sadly over the marble battlements at the Pennsylvania Station in the place marked "Inquiries". You know the Johnnies I mean. You go up to them and say: "When's the next train for Melonsquashville, Tennessee?" and they

110. Leigh HunT, The Indicator, vol. 1, 1820, p. 145.

111. " $[T]$ he vowel $[i],[\ldots]$ especially in it narrow or thin form, serves very often to indicate what is slight, insignificant, or weak. / La voyelle $<\mathrm{i}>,[. .$.$] particulièrement sous sa$ forme étroite ou grêle, sert très souvent à dénoter ce qui est frêle, insignifiant, ou faible. » Otto JESPERSEN, « Symbolic value of the letter I » [1922], dans Selected writings of Otto Jespersen, Abingdon, Routledge, 2010 [1960], p. 287.

112. Selon le dictionnaire historique d'Oxford, après le XVII' siècle, les créations lexicales faisant intervenir la désinence adjectivale $-\mathrm{Y}$ « tend in a large measure to be colloquial, undignified, or trivial, as bumpy, dumpy, flighty, hammy, liney, loopy, lumpy, lungy, messy, oniony, treey, verminy, vipery [... .] From the early years of the 19th cent. the suffix has been used still more freely in nonce-words designed to connote such characteristics of a person or thing as call for condemnation, ridicule, or contempt; hence such adjs. as beery, catty, churchy, jumpy, newspapery, piggy, tinny. / ont tendance dans une large mesure à être familières, relâchées, ou triviales, comme bumpy, dumpy, flighty, hammy, liney, loopy, lumpy, lungy, messy, oniony, treey, verminy, vipery [... .] $\dot{A}$ partir des premières années du XIX ${ }^{\mathrm{e}}$ siècle, le suffixe a été utilisé encore plus librement au sein de mots $a d$ hoc destinés à connoter les caractéristiques d'une personne ou d'une chose suscitant la réprobation, la dérision ou le mépris ; d'où les adjectifs comme beery, catty, churchy, jumpy, newspapery, piggy, tinny. » (C.-T. ONIONS, dans James A.H. Murray (dir.), A New English Dictionary on historical principles, Oxford, Clarendon Press, vol. X, part II, 1928, suppl. «X, Y, Z », p. 10).

113. « Johnny, Johnnie », New English dictionary, op. cit., vol. V, 1901, p. 592. 
reply, without stopping to think, "Two-forty-three, track ten, change at San Francisco ”. / Jeeves - mon valet, vous savez - est vraiment un type extraordinaire. Tellement compétent. Sans mentir, je serais perdu sans lui. Il est un peu comme ces types assis dans l'endroit marqué "Renseignements" à la gare centrale de New York, qui regardent par dessus le parapet de marbre d'un air morne. Vous voyez le genre de Johnnies dont je veux parler. Vous allez les trouver en demandant : “ $\mathrm{A}$ quelle heure est le prochain train pour Trou-en-cambrousse dans le Tennessee ?" et ils répondent, du tac au tac, "Quatorze heures quarantetrois, quai dix, prenez la correspondance à San Francisco" ${ }^{114}$. »

Se pliant aux règles de détermination et de pluralisation des noms communs, JOHNNY-quidam présentait alors une affinité particulière avec les déterminants indéfinis SOME et ANY exprimant l'incapacité/refus du locuteur de nommer l'objet de son discours ou de lui reconnaître une identité différenciée, ainsi qu'avec le démonstratif non-proximal THAT/ THOSE signifiant le rejet par l'énonciateur de cet objet dans la sphère du hors-Moi :

« Some Johnny - I forget just who - once said that "every soldier carries a Field Marshall's baton in his knapsack". Well, I don't know a lot about that yet, but I'm willin' to wager that there are a jolly sight more soldiers with a Boots "Economy Album" stowed conveniently in their Kit bags. / Un Johnny quelconque - son nom m'échappe - a déclaré que "tout soldat porte un bâton de maréchal dans sa giberne ${ }^{115}$ ". Ma foi, je n'y connais pas encore grand-chose, mais je suis prêt à parier qu'il y a bigrement plus de soldats qui ont un "Album économique" Boots à portée de main dans leur havresac ${ }^{116}$. »

«My dear Anthony, I find you merely ridiculous as a preacher, because you keep referring me to places and documents and alleged occurrences in which, as a matter of fact, I dont believe. [...] Your fishes and your catechisms and all the rest of it make a charming poem which you call your faith. [...] Who are you, anyhow, that you should know better than Mahomet or Confucius or any of the other Johnnies who have been on the job since the world existed? / Mon cher Anthony, vous faites un prédicateur proprement ridicule, parce que vous ne

114. P.-G. Wodehouse, My Man Jeeves, 1919, en ligne : http://www.gutenberg.org/ files/8164/8164-h/8164-h.htm.

115. Attribué au roi Louis XVIII par certaines sources françaises (cf. Le Légitimiste, 1833 1834, p. 197), cet aphorisme était prêté à Napoléon par la tradition britannique (cf. Household Words, 11 juil. 1857, p. 34).

116. The Amateur Photographer and Photographic News, Londres, Hazell, Watson \& Viney, vol. 66, 1917, p. 183. 
cessez de me renvoyer à des lieux, des documents et de supposés prodiges auxquels il se trouve que je ne crois pas. [...] Vos poissons, vos catéchismes et tout le tralala font de ce que vous appelez votre foi un charmant poème. [...] Qui êtes-vous, d'abord, pour prétendre savoir mieux que Mahomet ou Confucius ou n'importe lequel des autres Johnnies qui sont sur l'affaire depuis que le monde est monde ${ }^{117}$ ? »

" "Well”, returned the doctor agreeably. "We'll suppose the girls do look for an Englishman. Are we also to suppose that these young Boers, who haven't been sent down to the Cape for an education, are any more effusively fond of the English for that?" "Those Johnnies!" returned Morgan in amused contempt. "Doctor, those beggars don't count". / Eh bien, reprit aimablement le médecin, nous supposerons que les jeunes filles sont bien en quête d'un Anglais. Devons-nous également supposer que ces jeunes Boers, qui n'ont pas été envoyés au Cap pour y faire des études, en aiment les Anglais avec autant plus d'effusion ? - Ces Johnnies !, rétorqua Morgan, sur un ton de mépris amusé. Docteur, ces gueux ne comptent pas ${ }^{118}$. »

Opérateurs de distanciation par disqualification, JOHNNY, son alterego $\mathrm{JACK}^{119}$, et leur base commune JOHN entraient dans la composition d'une myriade de locutions classificatoires, de tonalité facétieuse ou injurieuse ${ }^{120}$ :

117. George Bernard SHAw, Getting married, a disquisitory play, Londres, Constable and Company, 1913 [1908], p. 290.

118. A.-O. VAughan, «The Girl with the soft grey eyes ", Longman's Magazine, vol. XV, nov. 1904-avr. 1905, p. 230.

119. « Jack. [...] 1. (as a proper name.) A familiar by-form of the name John [...] 2. (as a common noun.) A man of the common people ; a lad, fellow, chap ; esp. a low-bred, or ill-mannered fellow, a "knave". / Jack. [...] 1. (en tant que nom propre.) Une forme secondaire du nom John [...] 2. (en tant que nom commun.) Un homme du commun; un jeune homme, un gars, un type ; en particulier, un individu de basse extraction, ou malotru, un "maraud". » (New English dictionary, op. cit., vol. V, p. 533).

120. La première référence connue à William Shakespeare en tant que dramaturge raille la présomption sociale et culturelle de l'ex-acteur de province par le biais de la locution latine dont provient l'anglais JACK-OF-ALL-TRADES : "[T]here is an vpstart Crow, beautified with our feathers, that with his Tygers hart wrapt in a Players hyde, supposes he is as well able to bombast out a blanke verse as the best of you : and being an absolute Iohannes fac totum, is in his owne conceit the onely Shake-scene in a countrey. / Il y a un corbeau parvenu, embelli de nos plumes, qui, dissimulant son cœur de tigre sous une peau d'acteur, suppose qu'il est aussi capable que les meilleurs d'entre vous de boursoufler des vers; et qui, en parfait Johannes factotum, s'imagine dans sa vanité le seul branle-scène du pays. » (Robert GREENE, Green's Groats-Worth of Witte, Londres, 1592). 
«John-a-dreams, a dreamy fellow / John-des-rêves, un doux rêveur », «Johnny Raw [... ,] an inexperienced youngster, a new recruit / Johnny Mal dégrossi [..., ] un blanc-bec, une nouvelle recrue », « Jackgentleman, a man of low birth or manners, making pretensions to be a gentleman, an insolent fellow, an upstart / Jack-monsieur, un homme de basse extraction ou aux manières grossières, prétendant à la condition d'homme de bien, un impudent, un parvenu », "John Trot, a man of slow or uncultured intellect, a bumpkin, a clown / John Trot, un homme d'intellect lent ou non cultivé, un rustaud, un ballot», "Jack-out-ofdoors, a person turned out of his former place ; a homeless person, a vagrant / Jack-à-la-porte, une personne qui s'est vue chassée de son ancien domicile, un sans-logis, un vagabond » ${ }^{121}$, «John and Joan, an hermaphrodite / $\underline{\text { John }}$ et $\underline{\text { Joan, }}$ un hermaphrodite ", "John and Mary, a pair of country folk / John et Mary, un couple de gens de la campagne $\gg{ }^{122}$, etc. ${ }^{123}$.

Dans le sud-ouest de l'Angleterre, à une époque où l'appellation JOHNNY ONION était encore inconnue dans la région, les colporteurs autochtones étaient affublés d'un sobriquet catégorisant façonné sur un patron similaire $^{124}$ :

121. Tous les exemples cités jusqu'ici proviennent du dictionnaire historique d'Oxford.

122. Les deux derniers exemples sont tirés du monument lexicographique de Joseph WRIGHT, The English dialect dictionary, being the complete vocabulary of all dialect words still in use, or known to have been in use during the last two hundred years, vol. III, Oxford, Henry Frowde, 1905, p. 375-376.

123. L'abbé Feutren rappelait avec humour l'existence d'usages dénominatifs similaires dans la Basse-Bretagne de la fin du XIXe siècle : «L'année de la femme n'était pas encore passée par là [... et] il n'était [guère] respectueux pour les Marie de voir leur nom associé à des personnages tristement typés : MARI-PORC'H, une souillon, MARIFLAO, une femme sans ordre, MARI-MORGANT, une poissarde, [...] MARI JANNIG, une commère. Les hommes, il est vrai, n'étaient point épargnés, ni saint Jean mieux traité que Marie : YANN BANEZENN, Jean le Panais, un imbécile, YANN LAOU, couvert de poux, YANN-YANN, deux fois Jean, qui feint d'ignorer l'inconduite de sa femme, YANN-BILLENN, Jean guenille, YANN-IOUD, Jean bouillie, un benêt. Vivement l'année de l'homme !» (« Kresket ar zikour! », Bulletin paroissial de Roscoff, n² 297, été 1975, en ligne : http://www.roscoff-quotidien.eu/histoire-bulletin-paroissial-297.htm).

124. La désignation JOHNNY FORTNIGHT, attestée en Cornouailles dès 1863 (cf. «A Foreigner's view of Cornish life », The Dublin Evening Mail, 30 déc. 1863, p. 4), fut diffusée à l'échelle nationale dix ans plus tard par la publication dans le magazine populaire dirigé par Charles Dickens d'un récit éponyme (cf. «Johnny Fortnight », All the Year Round, nouv. série, ${ }^{\circ}$ 215, 11 juil. 1873, p. 199-203). Selon l'état actuel de mes recherches, il fallut attendre encore trente ans pour voir apparaître la désignation JOHNNY ONION dans un texte du sud-ouest de l'Angleterre (cf. « Suburban life », The Exeter and Plymouth Gazette, 18 nov. 1903, p. 3). 
«Few and far between are the excitements and novelties which enter into rustic life, though "Johnny Fortnight", as he is called in Devonshire, does indeed tramp the village street with regularity, and his packs of ribbons, trinkets, and fal-las furnish matter for feminine interest and excuse for wasting time at many wicket-gates. Other pedlars similarly trudge through the little centres of rural activity. During August, for instance, the picturesque onion boy shall be found limping in our desert places, and sometimes a little circus creeps through the hamlet [.] / Bien rares sont les attractions qui viennent rompre la monotonie de la vie rustique, bien que "Johnny Quinze Jours ${ }^{125}$ ”, comme on l'appelle dans le Devon, déambule tout de même régulièrement dans les rues du village, et que ses paquets de rubans, de colifichets et de falbalas y retiennent l'intérêt féminin et soient le prétexte de s'attarder à bien des barrières de ferme. D'autres marchands ambulants traversent pareillement les petits centres d'activité rurale. Courant août, par exemple, on trouve le pittoresque garçon-aux-oignons boitillant à travers nos déserts, et parfois un petit cirque traverse le hameau ${ }^{126}[$.$] »$

Les marchands d'oignons léonards cumulant la prévention sociale qui frappait les vagants et la défiance ethnique dont faisaient l'objet les populations non britanniques, il me paraît raisonnable de voir une motivation complémentaire du surnom JOHNNY ONION/ONION JOHNNY dans les dénominations xénophobes ou racistes construites sur le modèle JOHN(NY) + ethnonyme (ou attribut emblématique) qui servaient de faire-valoir à la figure allégorique nationale de John Bull ${ }^{127}$ en fustigeant l'ennemi ou rival du moment - Turcs pendant la Guerre de Crimée ${ }^{128}$, Chinois pendant les

125. Marchand à tempérament, le qualificatif «quinze jours » renvoyant au délai de paiement habituellement accordé aux clients des campagnes.

126. « Gundy Man », Black and White, 24 avr. 1897, p. 17.

127. Sur la genèse satirique du personnage de John Bull, voir Tamara L. HunT, Defining John Bull, Political caricature and national identity in late Georgian England, Aldershot, Ashgate, 2013.

128. «I am glad to say that none of the British have thus fallen into the hands of the Turks. There are, however, almost daily fights and broils between them and our soldiers and sailors. Yesterday a lot of men-of-war and merchant sailors came ashore for water. They remained at a small well until some Turkish bullock-drivers also came for water. The sailors having been first there would not give up the well, until "Johnny Turk" (as our men call them) began to push them about. Both parties argued the point (without understanding each other), but the Turks began to be impatient, and gave one of the sailors a push that sent him down a small brae. Instantly John Bull's blood was up [.] / Je me réjouis qu'aucun Britannique ne soit ainsi tombé aux mains des Turcs. Des bagarres et des querelles les opposent cependant presque quotidiennement à nos soldats et marins. Hier, de nombreux hommes de la marine militaire et marchande accostèrent pour se ravitailler en eau. Ils se trouvaient près d'un puits lorsqu' arrivèrent des bouviers 
Guerres de l'opium (illustr. 2), colons hollandais d'Afrique du Sud pendant la Guerre des Boers ${ }^{129}$, et surtout, depuis les guerres napoléoniennes, Français - :

« Il est très réel que les langoustiers de Camaret et de Paimpol était, dès avant la guerre, cordialement reçus, dans ces petits ports, comme les marchands d'oignons de Roscoff. Et puis, rapporte M. Allalo, "ils $\mathrm{y}$ achètent plus de sucre en huit jours que ne le feraient en un an les pêcheurs écossais". Des allumettes aussi. Les allumettes et le sucre faisaient qu'on les aimait bien, tout en riant. On riait encore de croire croyance répandue outre-Manche - qu'en vrais Français de France ils se nourrissaient habituellement de grenouilles (pas un pêcheur breton n'y goûterait) et de les surnommer en conséquence Johnny Crapaud. [...] Mais un Breton a-t-il besoin de sortir de France pour faire rire ou sourire ceux qui ne sont pas Bretons comme lui ${ }^{130}$ ? »

Ce double ostracisme, social et ethnique, se donne à lire et à voir sans ambages dans le premier texte et la première image (illustr. 3) où j'ai trouvé le vocable JOHNNY assigné aux marchands d'oignons léonards :

The Breton men and boys, with their monotonous cry of «Inyanns », are now looked for as regularly as autumn comes round, though it must be confessed that they are not quite so civil as they might be, nor as they used to be. Not content with standing in the streets, near the market, on a Saturday, they now perambulate the town and walk into private houses wherever they can find an open door, with a want of ceremony

qui venaient quérir de l'eau eux aussi. Les marins étant arrivés les premiers refusèrent de laisser la place, tant et si bien que les "Johnny Turc" (ainsi que les appellent nos hommes) commencèrent à les bousculer. Les deux parties affirmèrent leur bon droit (sans se comprendre), mais les Turcs finirent par s'impatienter, et poussèrent l'un des marins, qui dévala une petite pente. Le sang de John Bull ne fit qu'un tour [.] » (« Journal of a Highland Soldier in Turkey », The Inverness Courier, 14 sept. 1854, p. 6).

129. «Just a few lines to let you know that we are in the best of health. I received your letter all right, and I hope by the time you get this one we shall have taken Pretoria. We are still holding this position here, and Mister Johnny Boer thinks he is going to take it from us, but I think he is greatly mistaken [...] The Boer patrols occasionally come down to try and have a peep at what we are doing, but they never find John Bull's boys asleep [.]/ Voici seulement quelques lignes pour vous faire savoir que nous sommes en pleine santé. J'ai bien reçu votre lettre, et j'espère que lorsque vous recevrez celle-ci, nous aurons pris Pretoria. Nous tenons notre position. Monsieur Johnny Boer s'imagine qu'il va nous la prendre, mais je crois qu'il se trompe lourdement [...] Les patrouilles boer descendent parfois jusqu'ici pour tenter d'épier ce que nous faisons, mais jamais ils ne surprennent les troupes de John Bull à dormir [.] » (« Letter from Private Ford », The Bucks Herald, 16 juin 1900, p. 7).

130. « Pêcheurs bretons », La Revue de Paris, 15 mai 1919, p. 321. 


\title{
A CHANSON FOR CANTON.
}

\author{
Joms Chisamar a rogue is born, \\ The laws of truth he holds in scorn; \\ A bout as great a brute as can \\ Encumber the Earth is Jons Crinayax. \\ Sing YeIr, my cruel Joun Chinayan, \\ Sing Yeo, my stubborn John Cuinasan ; \\ Not COBDEN himself can take off the ban \\ By humanity laid on JoHs Chisaman.
}

With their little pig-eyes and their large piz-tails,

And their diet of rats, dogs, slugs, and snails,

All seems to be game in the frying-pan

Of that nasty feeder, JoIrn Cimsaman.

Sing lie-tea, my sly Jonn Curnamas,

No fightee, my coward Joun Cainaman :

Jons Bull has a chance-let him, if he can,

Somewhat open the ejes of Jorn Cuinaman.

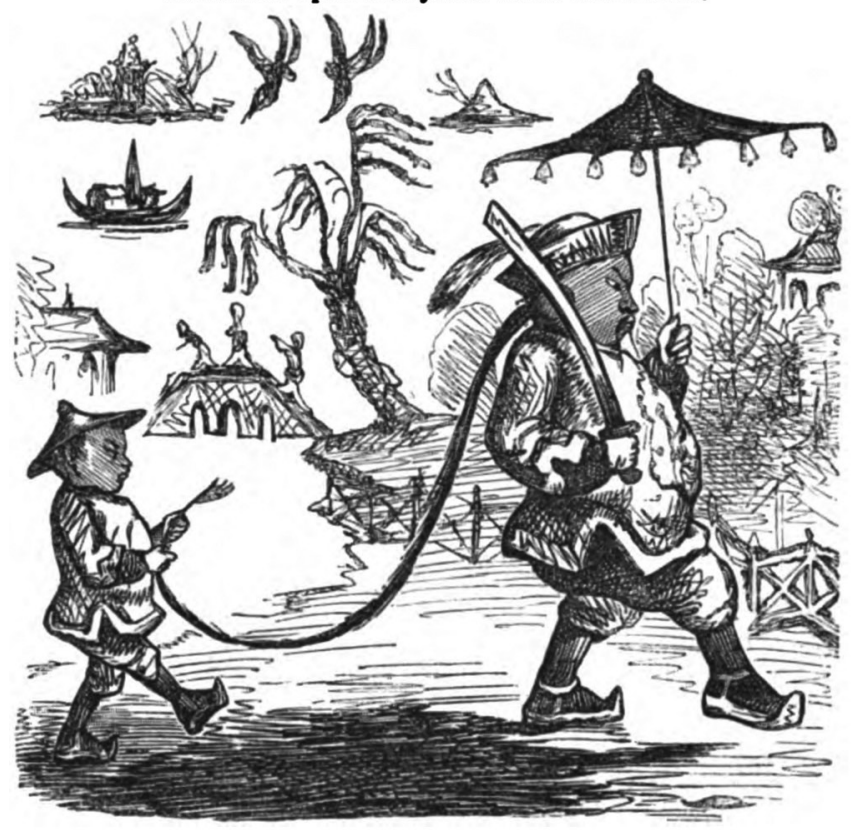

Illustr. 2 : " A Chanson for Canton », Punch, or the London Charivari, 10 avril 1858, p. 151.

that says little for their French manners. The way in which they handle door knockers is also far from pleasant to the nervous housekeeper. Some time ago, the borough authorities prosecuted some basket girls for selling vegetables in Roath, without having previously paid market toll. I should much like to know whether the "Onion Johnnies" pay toll before they start hawking the town? The persistent and annoying manner in which "Johnny" puts his feet between the door and the jamb, when a female answers his tremendous knock, and roars out 
"verra sheep ${ }^{131}$ " is quite startling to the nerves; but I have been led to say a few words about "Johnny " from a scene I witnessed on Saturday. The small boys of the town seem to consider "Johnny» as lawful prey for teasing, and about half-a-dozen youngsters were thus engaged on Saturday evening, when "Johnny" suddenly dropped his onions, ran after the biggest of his tormentors, and administered a kick with his heavy wooden sabot in the posterior of the unfortunate lad, which sent him howling and limping away. In fact, "Johnny» is far from being harmless and it is well it should be known that his temper is not to be trusted. I remember that last year one of them was sent to prison for kicking a woman violently on the leg. No one can justify the Cardiff boys being insolent to the onion men, but they, in turn, should be taught to use good language. The humbler class of foreigners seem to pick up "unmentionable» English words and they contrive to use them whenever they can't do as they wish - especially to females. Cannot Chief-constable Hemmingway read one or two a lesson? / Les hommes et garçons bretons, annoncés par leur cri monotone d'«Inyanns », réapparaissent désormais aussi régulièrement que s'en revient l'automne, bien qu'il faille avouer qu'ils n'observent guère les règles de civilité autant qu'ils le devraient ou le faisaient jadis. Non contents de stationner dans les rues, à proximité du marché, le samedi, ils pérambulent maintenant à travers la ville et s'introduisent dans les maisons particulières partout où ils trouvent porte ouverte, avec un manque de cérémonie qui fait peu honneur aux manières françaises. La façon dont ils manient les heurtoirs est elle aussi loin d'être plaisante pour la maîtresse de maison aux nerfs délicats. Il y a peu, la municipalité a poursuivi des filles-au-panier qui vendaient des légumes dans le quartier de Roath sans s'être préalablement acquittées de la taxe de droit de place. J'aimerais fort savoir si les " Onion Johnnies » paient quoi que ce soit avant d'entreprendre leur colportage ? La manière insistante et importune qu'a « Johnny » de mettre son pied entre la porte et le montant lorsqu'une personne de sexe féminin répond à ses coups de semonce, et de rugir " verra sheep ", met les nerfs à bien rude épreuve ; mais c'est la scène dont j'ai été témoin samedi dernier qui m'amène à dire quelques mots au sujet de "Johnny ». Les garçonnets de la ville semblent se croire autorisés à tourmenter " Johnny » impunément, et environ une demi-douzaine de polissons était ainsi occupée samedi soir

131. Présenté comme énonçant le principal argument commercial des marchands d'oignons léonards, l'adjectif « cheap » [bon marché] était fréquemment rendu sous la graphie « sheep ». Jouant sur l'homographie avec le nom « sheep » [mouton] - tout en singeant la difficulté des Bretons à restituer la dentale initiale de la séquence /t $\mathrm{fi}: \mathrm{p} /$-, ce procédé ressortit à un réseau de tropes qui associaient, ouvertement ou de façon plus insidieuse, les vendeurs d'oignons à l'animalité. 
lorsque « Johnny » posa soudain ses oignons, courut après le plus grand de ses tourmenteurs, et administra un coup de son lourd sabot dans le postérieur du jeune infortuné, qui s'en fut en hurlant et boitant. En fait, " Johnny » est loin d'être inoffensif et c'est une bonne chose que l'on sache devoir se méfier de ses humeurs. Je me rappelle que l'année passée, l'un d'entre eux fut envoyé en prison pour avoir violemment frappé une femme à la jambe. Nul ne peut justifier l'insolence dont les garçons de Cardiff accablent les hommes-aux-oignons, mais ces derniers devraient, en retour, se voir apprendre à employer un langage correct. Ces étrangers des classes humbles semblent attraper des mots anglais « inmentionnables » et trouver moyen d'en faire usage à chaque fois qu'ils ne peuvent parvenir à leurs fins - particulièrement auprès des femmes. Le commissaire Hemmingway ne pourrait-il pas faire la leçon à un ou deux d'entre eux ${ }^{132}$ ?

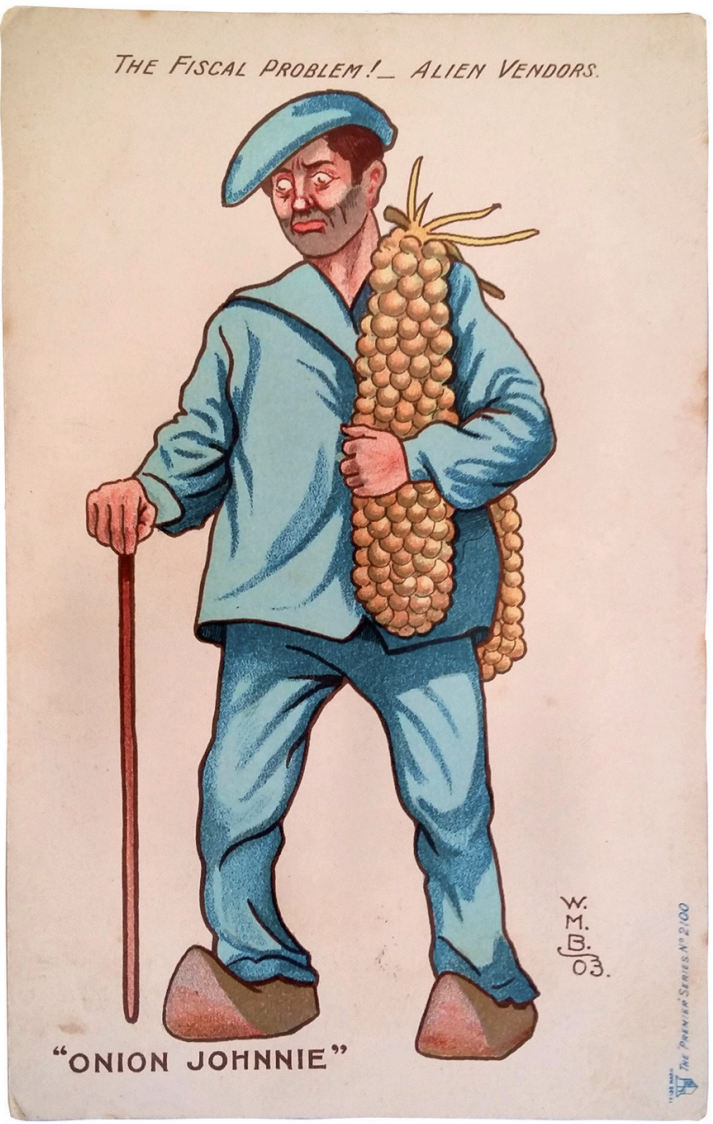

Illustr. 3 :

Carte postale anglaise éditée en 1903

(coll. E. Boudillet).

132. Peter Playfair, «Echoes of the week», The Cardiff Times, 17 nov. 1877, p. 8. 
De nos jours, lors de la messe en plein air qui constitue chaque été le point culminant du pardon de Roscoff, le chœur des paroissiens ranime, le temps d'un cantique, le souvenir du déracinement cyclique des marchands d'oignons, présentés comme des sujets parlants dont la parole mérite considération :

Écoutez dans le vent,

La voix de nos johnnies,

Ils ont quitté leurs champs

Leur prêtre les bénit (bis)

À Sainte Barbe, au pied de la chapelle (bis)

Allié aux puissants marqueurs d'intégration que sont les déterminants possessifs («nos johnnies », «leur prêtre »), et mis à la rime avec le verbe performatif «bénit», le nom JOHNNY a désormais rejoint le registre du sacré (illustr. 4).
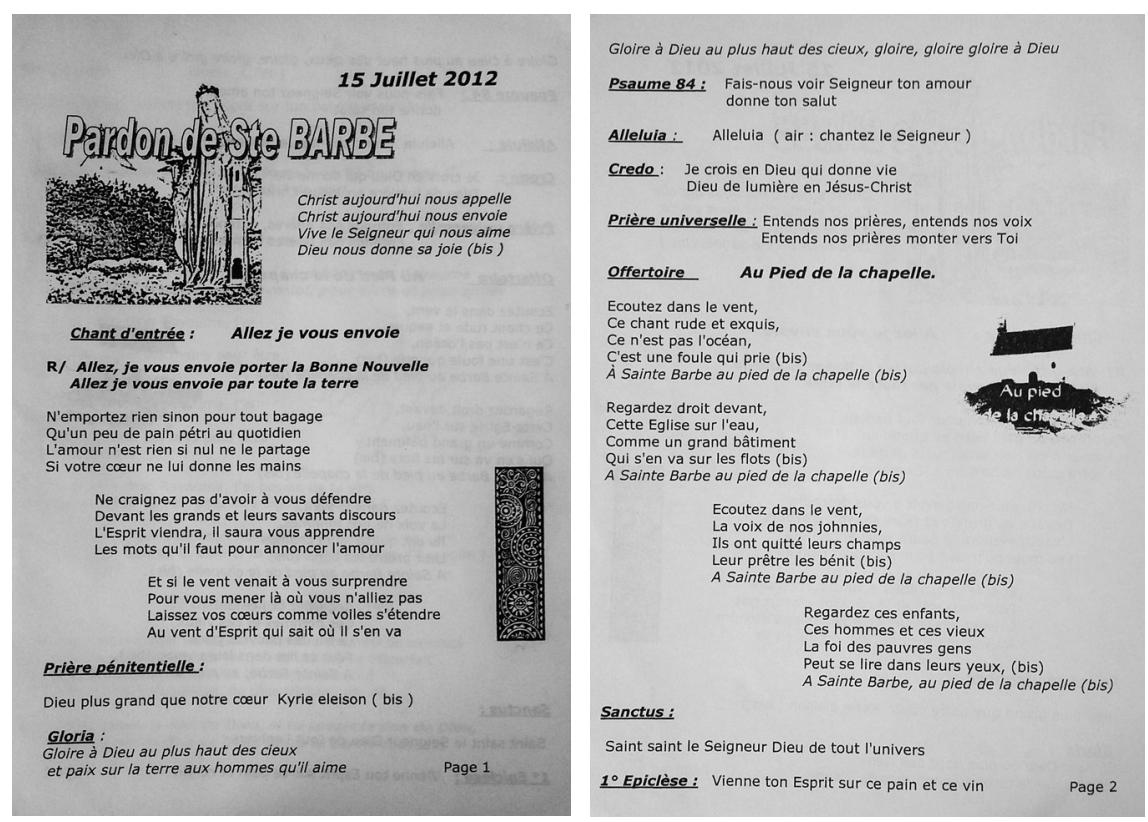

Illustr. 4 : Feuille de chants du pardon de Sainte Barbe, Roscoff, 15 juillet 2012, p. 1-2. 
Pour opérer cette conversion ${ }^{133}$ quasi miraculeuse, cet « acte de magie sociale ${ }^{134}$ » instituant «en tant que groupe connu et reconnu ${ }^{135}$ » une classe d'individus longtemps tenue pour « innommable ${ }^{136} »$, il aura fallu la détermination, l'éloquence, et, par dessus tout, la profonde humanité d'un « Jean des Merveilles », dont la position d'entre-deux fit de lui un passeur de mots.

Fils de marchands de légumes et prix d'excellence au Collège du Kreisker, héros-mutilé de guerre et prêtre jociste, sillonnant le Finistère à mobylette dans sa soutane doublée de papier journal, François-Yves Creignou ${ }^{137}$ fit paraître, entre 1927 et 1937, dans le Courrier du Finistère d'abord, puis dans la Voix de Sainte Barbe ${ }^{138}$, plusieurs dizaines de textes bretons ou français, anonymes pour la plupart. Au travers de ces reportages, vignettes et historiettes, tour à tour didactiques, pathétiques ou comiques, le futur vice-postulateur de la cause en béatification de Michel Le Nobletz procéda à la transmutation de JOHNNY de mot-repoussoir en motostensoir, faisant ainsi sortir les marchands d'oignons léonards des limbes symboliques dans lesquelles ils étaient d'ordinaire tenus jusqu'alors.

Et répondant, d'une certaine façon, à l'appel solitaire lancé, quelque quarante ans plus tôt, depuis l'autre rive de la Manche, par l'épouse d'un pasteur protestant :

"I wish to lay the case of these boys on the hearts and conscience of my sisters. Where are the merry girls whose French is so ready

133. "Naming is a crucial aspect of converting "anybodies" into "somebodies" / La nomination joue un rôle crucial dans la conversion des "on-ne-sait-qui" en "quelqu'un". » Clifford GeERTZ, The Interpretation of culture, New York, Basic Books, 1973, p. 363, cité par Sophie CHAVE-DARTOEN, « Noms propres, catégorisation, sémantique et rapport au monde », dans Nomination et organisation sociale, Sophie Chave-Dartoen, Cécile Leguy et Denis Monnerie (dir.), Paris, Armand Colin, coll. Recherches, 2012, p. 122.

134. P. Bourdieu, Ce que parler veut dire, L'Économie des échanges linguistiques, Paris, Fayard, 1982, p. 125.

135. Ibidem, p. 142.

136. Ibid.

137. Né à Roscoff le 25 novembre 1889 sous le nom de «François Creignou », le doyen des prêtres du Finistère fut inhumé dans le caveau familial le 13 juin 1981 sous celui d' « Yves Creignou », ayant choisi, après son ordination en 1920, de porter le prénom de son parrain marchand d'oignons. Les « Marvailhou an Amzer Goz » qu'il consacra aux « Johnniget» sont signés des initiales « Y.C. ».

138. Bulletin paroissial de Roscoff, dont le premier numéro parut le 15 mars 1934. Dès l'automne suivant, F.-Y. Creignou y publia une «Pajen ar Johnniget », signée «Ho mignoun fidel. Y.C. ». Je tiens à remercier du fond du cœur Anne-Marie Creignou, qui a eu l'immense gentillesse de me confier sa collection de Voix de Sainte Barbe - la seule, semble-t-il, a avoir été préservée jusqu'à aujourd'hui. 


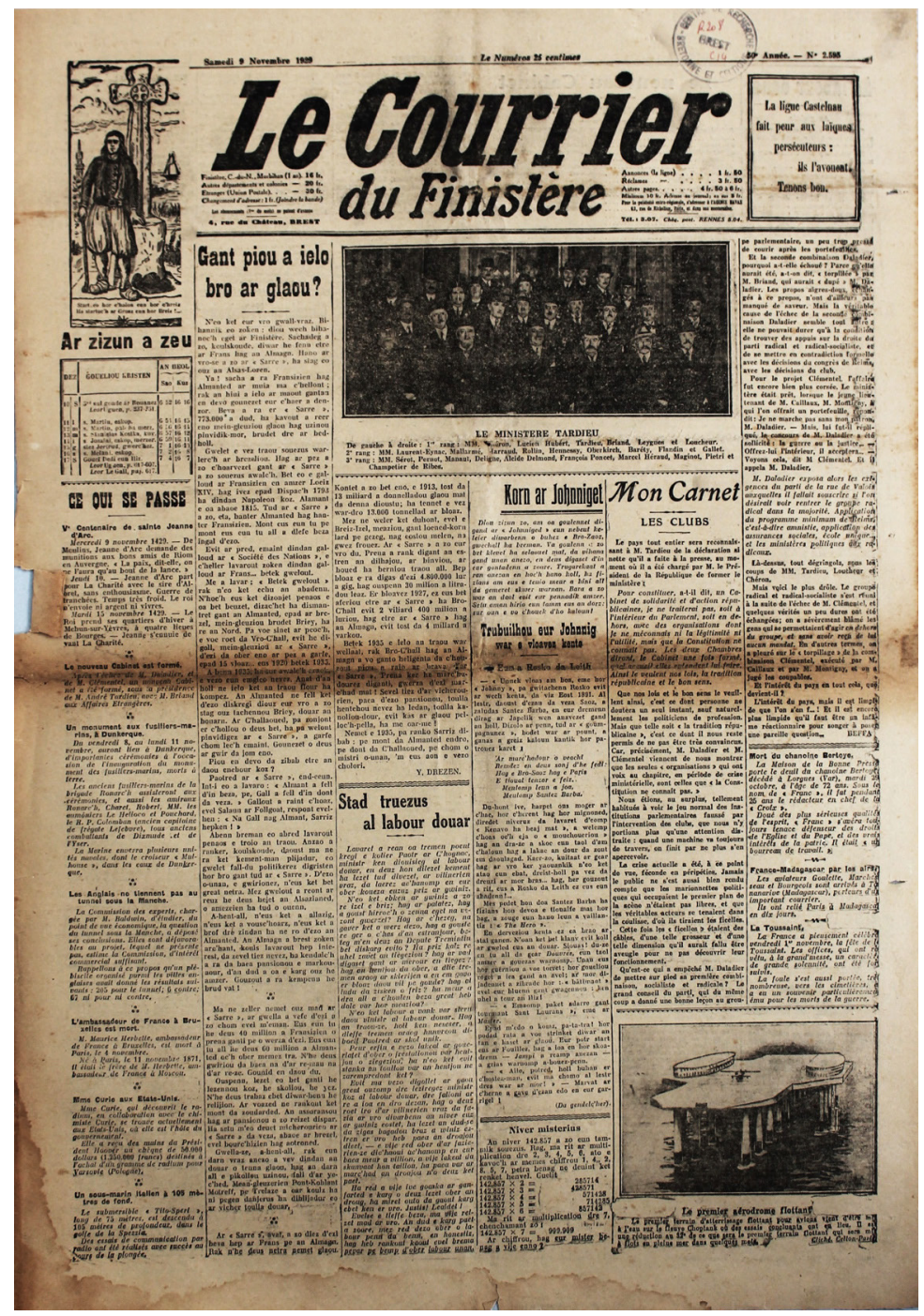

Illustr. 5 : Page de Une du Courrier du Finistère du 9 novembre 1929 (coll. CRBC). La parution de l'épisode inaugural du feuilleton "Trubuilhou eur Johnnig war e vloavez kenta » marque l'apogée du processus de démarginalisation symbolique des marchands d'oignons léonards, dont la dénomination bretonnisée se voit placée, littéralement, au centre de l'attention.

and fluent? Have they not a word of cheer for the poor tired onion boy? Where is the cultivated Christian lady, whose sympathies are always so ready and whose hand is ever prompt to help? Speaking, the other Sunday, of God's power, and saying that with Him, nothing is impossible, one of my boys looked up and said: "One thing is 
impossible, madame", "What is that?" I asked. "Paradise for us!" Oh that the time may come, when by the kind and loving teaching of Christian people in all the ports to which they come, Paradise may be opened to these poor Bréton [sic] lads, that they may be no longer "strangers and foreigners, but fellow-citizens with the saints, and of the household of God"! / Je souhaite soumettre le cas de ces garçons au cœur et à la conscience de mes sœurs. Où sont les jeunes filles enjouées dont la connaissance du français n'attend que d'être mise à profit? N'ont-elles point un mot de réconfort pour redonner courage au pauvre garçon-aux-oignons fatigué ? Où est la chrétienne cultivée, dont la compassion est toujours prête à s'émouvoir et la main toujours prompte à secourir ? Comme j'évoquais, l'autre dimanche, la puissance de Dieu, rappelant que rien ne Lui est impossible, l'un de mes garçons leva les yeux et s'écria "Il y a une chose qui est impossible, madame", "De quoi s'agit-il ?" demandai-je. "Le Paradis pour nous autres !" Ô que vienne le temps où, grâce à l'enseignement dispensé avec bonté et amour par les chrétiens de tous les ports qu'ils fréquentent, le Paradis s'ouvrira à ces pauvres garçons bretons, afin qu'ils ne soient plus "des étrangers et des gens du dehors, mais des concitoyens des saints et des gens de la maison de Dieu" "139! » 


\section{Balises chronologiques (1815-1939)}

NB : Il ne saurait être question de dresser ici un tableau exhaustif ou définitif des temps forts de l'histoire factuelle et symbolique des marchands d'oignons léonards ${ }^{1}$. Sauf mention contraire, tous les jalons composant ce canevas provisoire s'appuient sur mes recherches en cours et sont appelés à être révisés, précisés et complétés au fur et à mesure de la mise au jour de nouvelles sources documentaires. Ne prétendant aucunement remonter à un hypothétique point d'origine absolu, chacune des «premières » occurrences ou manifestations répertoriées cidessous s'entend donc prudemment au sens tout relatif de plus ancienne apparition attestée par la documentation dont j'ai connaissance à ce jour.

\begin{tabular}{|c|c|}
\hline 1815 & $\begin{array}{l}\text { Reprise progressive du trafic commercial licite entre le port de Roscoff et la Grande-Bretagne après } \\
\text { la levée du Blocus continental. } \\
5 \text { passeports à destination de Plymouth/Falmouth sont délivrés par la municipalité de Roscoff à de } \\
\text { jeunes « cultivateur[s], marchand[s] de légumes ». }\end{array}$ \\
\hline 1816 & $\begin{array}{l}\text { Les colonnes commerciales d'un quotidien régional britannique consignent deux arrivées de } \\
\text { cargaisons d'oignons en provenance de Roscoff dans le port de Plymouth. Une nouvelle entrée est } \\
\text { signalée en } 1819 \text {, suivie d'un hiatus de près de } 30 \text { ans : on ne retrouve ensuite mention d'oignons } \\
\text { importés de Roscoff qu'en 1848, dans le nord industriel du pays (Newcastle). }\end{array}$ \\
\hline 1828 & $\begin{array}{l}\text {-Année retenue par la tradition populaire, littéraire et historiographique bretonne comme constituant } \\
\text { le point de départ de l' « épopée » ou « saga » des « Johnnies », bien qu'aucune donnée archivistique } \\
\text { découverte pour l'heure ne permette d'en étayer l'historicité }{ }^{2} \text {. } \\
\text {-1re reconnaissance officielle de la profession de « marchand d'oignons » en pays de Léon, au sein } \\
\text { d'un acte de naissance dressé à Roscoff }{ }^{3} \text {. La dénomination traverse ensuite une longue période } \\
\text { de dormance, ne resurgissant que trois décennies plus tard, lors de la transcription en mairie de } \\
\text { Roscoff de l'acte de décès d'un saisonnier roscovite ayant trouvé la mort outre-Manche (Plymouth, } \\
\text { oct. } 1858)^{4} \text {. }\end{array}$ \\
\hline 1848 & $\begin{array}{l}\text { Plus ancienne trace parvenue jusqu'à nous, dans les registres d'immigration britanniques, du } \\
\text { débarquement outre-Manche de "merchant[s]»/ «farmer[s] " partis de Roscoff (Plymouth, } \\
3 \text { voyageurs concernés). }\end{array}$ \\
\hline 1858 & $\begin{array}{l}1^{\text {re }} \text { vague migratoire significative constatée par les archives maritimes bretonnes : entre juillet et } \\
\text { septembre, plus de cent « cultivateurs » léonards s'embarquent « pour affaires de commerce » à } \\
\text { bord de bateaux chargés d'oignons en partance pour l'Angleterre et le pays de Galles }{ }^{5} \text {. }\end{array}$ \\
\hline 1859 & $\begin{array}{l}\text { 1re allusion explicite, au moyen d'une désignation périphrastique, aux « Frenchmen who went about } \\
\text { the streets selling onions / les Français qui parcouraient les rues pour vendre des oignons » dans } \\
\text { un article de presse dénonçant l'iniquité supposée de la politique libre-échangiste récemment initiée } \\
\text { par le Royaume-Uni (Exeter). }\end{array}$ \\
\hline
\end{tabular}

1. Je n'ai retenu dans mon tableau que les événements et processus me paraissant susceptibles d'éclairer ou de mettre en perspective l'avènement du mot JOHNNY comme surnom des marchands d'oignons léonards. En complément, on pourra se reporter aux deux études panoramiques menées au moment du crépuscule de la profession : François Guivarch, Les Johnnies de Roscoff, Quimper, Nature et Bretagne, 1979 [1969-1970], et Jean-Jacques Moncus, L'Émigration saisonnière des Johnnies de Roscoff des origines à nos jours, mémoire de maîtrise, Brest, UBO, 1973. 
-Un « French onion seller » apparaît pour la 1 re fois dans la rubrique des faits divers d'un quotidien régional britannique (Newport, pays de Galles). Dès lors, et sur l'ensemble de la période étudiée, les pages des faits divers constituent le cadre discursif le plus fréquenté par les marchands d'oignons léonards - que ces derniers y revêtent le statut de victimes ou d'accusés. (Plus de 200 textes répertoriés au sein du corpus britannique.)

1866 -Le pasteur-voyageur Samuel Prideaux Tregelles évoque, en marge du récit d'une mission évangélique en Bretagne, "the Breton onion-sellers, who, as usual, have come to this city of Plymouth in large parties / les vendeurs d'oignons bretons, qui, comme à l'accoutumée, sont arrivés dans notre ville de Plymouth en grands groupes ». Dans cette région de Grande-Bretagne située immédiatement à l'aplomb de Roscoff, le cycle migratoire des marchands d'oignons léonards fait ainsi figure, dès le milieu des années 1860, de phénomène installé, à la fois régulier et relativement massif.

Alexandre Dumas père, à la faveur d'une villégiature roscovite, rédige l'article « Oignon » de son Grand dictionnaire gastronomique (1873).

1869 Le récit de fondation rocambolesque qu'il raconte avoir recueilli auprès de l'armateur Édouard Corbière situe l'expédition originelle dans la capitale londonienne, mais ne précise pas la date à laquelle son héros solitaire et anonyme aurait initié le négoce des oignons avec la Grande-Bretagne.

-Plus ancienne référence expresse au " pardon de sainte Barbe », dans une lettre du peintre JeanLouis Hamon écrite lors d'un séjour à Roscoff chez son ami Tristan Corbière. Coïncidant avec le moment du départ annuel des marchands d'oignons, cette manifestation estivale, à la fois religieuse et festive, sera plus tard connue pour être «le pardon des " partants » ».

-Le «French Onion Boy » vient s'inscrire au répertoire d'imitations burlesques des chansonniers anglais (Newcastle).

-Au lendemain de la chute du Second Empire, un article de presse britannique imagine l'empereur déchu « disguised as a Normandy sailor / déguisé en marin normand » et ralliant les côtes anglaises à bord d'un sloop chargé d'oignons (Newcastle). Ce détournement parodique à des fins de satire politique, qui érige la figure du marchand d'oignons (léonard, mais non perçu comme tel) en représentant archétypal du Français, demeure un topos vivace dans la Grande-Bretagne du début du XXIe siècle 6 .

1875- Le périmètre d'activité des Léonards s'étend à l'Écosse, ceinturant désormais toute la Grande1880 Bretagne.

1882 Un « French onion seller » est signalé comme sujet pictural pour la 1re fois lors d'une exposition de peinture anglaise (Exeter).

Pierre des Jars de Keranroué, membre de la Section d'agriculture de la Breuriez Breiz, fait paraître dans le Courrier du Finistère un long reportage vantant le dynamisme des maraîchers léonards, dans le but de proposer le modèle " roscovite » en contre-exemple à l'ensemble d'une Bretagne 1886 rurale saignée par un exode définitif galopant.

Cette enquête constitue en outre la seule tentative connue de chiffrer et situer les marchands d'oignons présents en Grande-Bretagne à la fin du XIXe siècle : 451 patrons et employés, regroupés au sein de 26 « compagnies » basées dans 13 villes anglaises, galloises et écossaises.

-Durant leurs séjours outre-Manche, les marchands d'oignons léonards se voient distribuer des textes et chants religieux en breton par l'association missionnaire protestante « the Bible Society ». A Liverpool, des pasteurs gallois (baptistes et méthodistes) œuvrent activement auprès des " gwyry winwyns / hommes-aux-oignons », en lesquels ils voient des vecteurs de conversion en puissance,

1880- à même de propager efficacement - et gratuitement - la foi protestante en terre bretonne lors de 1890 leur retour annuel au pays.

-À compter des années 1890, sauf rarissimes exceptions, toute mention de colporteurs d'oignons dans les sources britanniques renvoie systématiquement et exclusivement aux migrants léonards, qui semblent alors avoir entièrement supplanté les vendeurs indigènes encore signalés, mais de plus en plus sporadiquement, au cours des décennies précédentes. 


\begin{tabular}{|c|c|}
\hline 1895 & $\begin{array}{l}\text {-La presse britannique entreprend de véritables reportages de terrain sur les marchands d'oignons } \\
\text { léonards, ainsi institués en objet discursif à part entière. } \\
\text {-Entrée en littérature de la figure du marchand d'oignons breton, dans les pages d'un roman } \\
\text { sentimental didactique rédigé en dialecte du Somerset (mais paru à Londres et diffusé jusqu'en } \\
\text { Écosse). }\end{array}$ \\
\hline 1896 & $\begin{array}{l}\text { Ouverture à Roscoff d'un débit de boisson tenu par un marchand d'oignons. Perpétué par les fils et } \\
\text { petit-fils du fondateur, eux aussi tenanciers/marchands d'oignons, ce centre de convivialité où l'on } \\
\text { vient boire une « païntad » de bière, jouer aux « dartze » [fléchettes anglaises (darts)] et où l'anglais } \\
\text { se mêle au breton et gallois, prendra dans les années 1960-1970 le nom de « Bar des Johnnies ». }\end{array}$ \\
\hline 1898 & $\begin{array}{l}\text { Naufrage du Channel Queen, au cours duquel } 14 \text { marchands d'oignons léonards trouvent la mort } \\
\text { (Guernesey, } 1 \text { er-2 février). } \\
\text { Relayée par les rédactions britanniques, bretonnes et françaises, la catastrophe révèle l'existence } \\
\text { des marchands d'oignons bretons au-delà de leur terroir d'origine et des sphères où s'exerce leur } \\
\text { commerce. }\end{array}$ \\
\hline 1899 & $\begin{array}{l}\text {-Le boycott orchestré par la presse britannique en protestation contre l'Affaire Dreyfus affecte } \\
\text { sporadiquement le négoce des Léonards. } \\
\text {-A l'occasion de l'Eistedfodd de Cardiff, est évoquée la proposition d'orner la « Grande Bannière du } \\
\text { Gorsedd » de tresses d'oignons afin de symboliser « l'union du pays de Galles et de la Bretagne ». }\end{array}$ \\
\hline c. 1900 & $\begin{array}{l}\text { En Angleterre et au pays de Galles, la silhouette exotique du marchand d'oignons léonard vient } \\
\text { s'intégrer à l'imagerie pittoresque des cartes postales. }\end{array}$ \\
\hline 1904 & $\begin{array}{l}\text { Fondation par le recteur de Roscoff de la «Société de Sainte Barbe », en réaction contre les } \\
\text { initiatives caritatives et pastorales d'églises protestantes britanniques en direction des marchands } \\
\text { d'oignon léonards. Intervention du député Albert de Mun auprès des autorités et notabilités } \\
\text { catholiques insulaires à leur sujet. }\end{array}$ \\
\hline 1905 & $\begin{array}{l}\text {-En Grande-Bretagne, l'image visuelle et textuelle du marchand d'oignons français est régulière- } \\
\text { ment invoquée en tant que personnification de l'« Entente Cordiale ». } \\
\text {-Le naufrage du Hilda (Saint-Malo, 18-19 nov.), dans lequel sombre une centaine de marchands } \\
\text { d'oignons, fait l'objet d'une intense couverture médiatique, dont la vaste portée spatio-temporelle } \\
\text { confère aux migrants léonards une notoriété et une visibilité inédites. Parmi les très nombreux } \\
\text { textes (discours, lettres, articles, complaintes, poèmes, etc.) suscités par le drame, seule une courte } \\
\text { nouvelle britannique destinée à un lectorat enfantin emploie la dénomination JOHNNY. }\end{array}$ \\
\hline 1910 & $\begin{array}{l}\text { - Constitution à Roscoff, à l'instigation de « commerçants » et « marchands d'oignons », de la } \\
\text { "Société mutuelle accidents cultivateurs-vendeurs d'oignons de Roscoff », rebaptisée « Mutuelle } \\
\text {-Retraite spirituelle réservée aux « "partants" pour l'Angleterre » organisée à l'initiative du recteur } \\
\text { de St-Pol-de-Léon avant l'exode annuel. Compte rendu diffusé par la Semaine religieuse de } \\
\text { Quimper et Léon. }\end{array}$ \\
\hline 1912 & $\begin{array}{l}\text { À Douarnenez, lors du Congrès de la Fédération régionaliste de Bretagne, le jeune Francis } \\
\text { Gourvil donne la } 1 \text { re audition publique du chant « Paotred Rosko » devant une assistance mêlée } \\
\text { d'intellectuels, de pêcheurs et de sardinières. Écrit par le " barde » et notable roscovite Eugène } \\
\text { d'Herbais sur une mélodie galloise, ce texte à succès }{ }^{9} \text { exalte l'intrépidité des vendeurs-voyageurs } \\
\text { bretons sans recourir à la dénomination JOHNNY. }\end{array}$ \\
\hline 1914 & $\begin{array}{l}\text { Le missionnaire baptiste } \mathrm{C} \text {. H. Jenkins, en poste à Morlaix, appelle ses confrères restés au pays de } \\
\text { Galles à travailler à la conversion des marchands d'oignons bretons. }\end{array}$ \\
\hline 1920 & $\begin{array}{l}\text { En Grande-Bretagne, le retour des marchands d'oignons léonards après la coupure de la Grande } \\
\text { Guerre est salué comme la reprise du cours naturel de la vie. }\end{array}$ \\
\hline
\end{tabular}


-Le Courrier du Finistère appelle " la petite patrie " à commémorer le centenaire putatif de la fondation du « trafic anglo-breton ».

1927

-La London Teachers'Association diligente une enquête sur le sort des « French children of school age [working] as peripatetic vendors of onions / enfants en âge scolaire travaillant comme vendeurs d'oignons péripatétiques » visant à faire expulser du territoire britannique les vendeurs les plus jeunes.

-La presse britannique vulgarise le mythe de l'intercompréhension « naturelle » du migrant breton et

1925- de ses interlocuteurs " celtes » (galloisants et supposés cornicophones).

1930 -Les pages féminines des journaux britanniques s'approprient une figure solarisée et érotisée du marchand d'oignons, qu'elles investissent d'un fort potentiel d'évasion.

-Acmé probable du commerce trans-Manche des oignons : environ 1300 hommes de la région de Roscoff sillonneraient les routes britanniques ${ }^{10}$.

-Nombre record de textes britanniques et bretons faisant mention des marchands d'oignons léonards pour une année donnée (hors naufrage du Hilda).

-Instauration, en $1^{\text {re }}$ puis $2^{\mathrm{e}}$ page du Courrier du Finistère, d'une rubrique dédiée aux marchands d'oignons, intitulée "Korn ar Johnniget », dont les moments saillants sont notamment :

1929 --- un article métalinguistique en breton se proposant d'expliquer l'origine et le sens du vocable JOHNNY - cet essai sera repris et transposé en français dans le bulletin paroissial de Roscoff, la Voix de Ste Barbe, en 1934, sous la signature "Y.C. ».

--- les 15 épisodes du feuilleton anonyme "Trubuilhou eur Johnnig/y ${ }^{11}$ war e vloavez kenta " ${ }^{12}$, que Naïg Rozmor attribuera au marchand d'oignons roscovite Henri Corre (1879-1969) lors de sa réédition du récit sous forme de livret en 1988.

--- le chant « Good onions very cheap / Gwerz ar Johniget » de l'abbé Augustin Conq, ancien vicaire de St-Pol-de-Léon (1899-1920) ${ }^{13}$.

-En Grande-Bretagne comme en Bretagne, le médium radiophonique se saisit du personnage du marchand d'oignons léonard - héros de pièces de théâtre ou chanteur de mélodies réputées « traditionnelles » - et lui confère un rayonnement sans précédent.

années

-Résurgence dans la littérature populaire britannique de la représentation du « foreign onion seller » comme archétype de l'étranger inquiétant : vociférant un sabir barbare, il est sale et malodorant, brun et basané, païen ou " papiste » ; associé au grouillement du rat et de la vermine, il cristallise les peurs de l'invasion et de la contamination, du vol et du viol.

-Le photographe d'origine galloise Angus McBean, figure phare du courant surréaliste londonien et portraitiste des stars du théâtre et du cinéma, fait poser un « Basque onion boy », derrière lequel on devine un marchand d'oignons léonard.

1937 -Un marchand d'oignons léonard participe au programme télévisuel « Picture Page », diffusé par la BBC ${ }^{14}$ le samedi soir à l'heure de grande audience. Le compte-rendu de l'émission publié dans le magazine Radio Times donne du « Breton onion boy » l'image d'un Bon Sauvage des temps modernes.

-Soutenue par les autorités locales et l'Union des CEuvres bretonnes, la « Marche des Johnnies » de St-Pol-de-Léon bénéficie d'une véritable campagne de presse conduite concurremment par les deux grands quotidiens locaux, la Dépêche de Brest et l'Ouest-Éclair.

-En préparation de la visite d'état du Président Lebrun à Londres, la BBC se déplace à Roscoff afin d'y enregistrer le « message d'amitié » des marchands d'oignons français au peuple britannique.

1939 -A la veille de l'entrée en guerre, Francis Gourvil rédige pour l'édition dominicale de l'Ouest-Éclair un reportage-fleuve abondamment illustré, au titre-programme : « Johnny le Roscovite, dit « Le BellBreaker », approvisionne notre ami John Bull en oignons léonards » (6 sept.)

-Peu après l'éclatement du conflit, paraît en 1 re page du Courrier du Finistère un cantique à Ste Barbe signé Laouenanig Breiz, nom de plume du frère Visant Séité, alors directeur de l'école SteBarbe de Roscoff. Sous la forme « Joniged Rosko », le vocable JOHNNY connaît alors son ultime consécration en accédant au lexique des chants religieux ( 28 oct.). 


\section{Notes}

2. Voir l'enquête conduite par Dany Guillou-Beuzit : « Henri Ollivier, premier Johnny?», art. cit.

3. Alors que la naissance survient en automne, haute saison du commerce des oignons, le père de l'enfant - frère cadet d'un des cinq passagers pour Plymouth de 1815 et cousin germain par alliance d'Henri Ollivier - est présent au moment de la déclaration. Peutêtre se contente-t-il d'expédier sa marchandise sans l'accompagner ? Rien n'indique que son négoce concerne la Grande-Bretagne, mais si tel est néanmoins le cas, on peut imaginer qu'il effectue seulement de brefs séjours outre-Manche, vendant sa cargaison en gros à son arrivée sans en assurer lui-même la distribution au détail, en accord avec le mode opératoire décrit dans les premiers textes agronomiques à faire état de l'exportation d'oignons du Léon en Grande-Bretagne, à partir de 1845. (Je remercie Jean-Pierre Le Saint d'avoir attiré mon attention sur ce précieux document.)

4. Je remercie Dany Guillou-Beuzit de m'avoir signalé cette transcription.

5. Je me repose ici sur le minutieux travail d'inventaire effectué par Jean-Marie Balanant à partir des registres des sorties de bâtiments conservés au Service Historique de la Marine de Brest et des registres des passeports consultables aux Archives départementales du Finistère.

6. En témoigne par exemple la déclaration du Secrétaire d'État aux Affaires étrangères à propos du traité de coopération militaire franco-britannique signé à l'automne 2010 : "We're not merging our Army with France. Our soldiers won't be required to speak French or wear onions round their necks [...] or ride bicycles. / Il n'est pas question de fusionner notre Armée avec la France. Nos soldats n'auront pas à parler français, porter des oignons autour du cou [...] ou se déplacer à bicyclette. » (« Heckler brands minister racist on TV over French Army », BBC News, 4 nov. 2010, en ligne : http://www.bbc. co.uk/news/uk-politics-11699172).

7. La déferlante de reportages qui traduisit et alimenta l'onde de choc émotionnelle ressentie au lendemain du désastre ne retomba progressivement qu'à la toute fin de l'hiver 1906. Pour la seule sphère anglophone, la carte des articles de presse se faisant l'écho du naufrage englobe toutes les Îles Britanniques - zones non touchées directement par l'activité des marchands d'oignons y compris -, les quatre coins de l'Empire - du Canada à Singapour en passant par les Bermudes, la Nouvelle-Zélande ou l'Australie, et l'ensemble des États-Unis - de New York à San Francisco, de Saint-Louis à Salt Lake City. Michèle Segura-Coz, présidente de l'association « Le Hilda », a par ailleurs répertorié des articles allemands, espagnols, italiens, néerlandais... et mexicains.

8. Je remercie Jean-Marie Balanant d'avoir porté à ma connaissance la brève existence de cette association.

9. À l'été 1916, le chant «Paotred Rosko » était déjà auréolé d'une notoriété telle qu'il fut choisi comme support de la souscription lancée par F. Gourvil dans le cadre de l'« Euvre de la Chanson bretonne » afin d'« égayer et réconforter toute l'armée et la marine bretonne $»$. Véritable tube identitaire auprès de publics variés voire antagonistes, cet « hymne national » (Jean Feutren) trouva place aussi bien dans les pages de la revue catholique Feiz ha Breiz (déc. 1919 par ex.) que sur la bande-son du film Breiz nevez, tourné à Pont-l'Abbé en août 1938 lors d'une grande fête folklorique organisée par le Parti communiste et l'association des Bretons émancipés menée par Marcel Cachin (film visionnable sur le site du Fonds audiovisuel du PCF, à l'adresse http://www. cinearchives.org/Films-447-61-0-0.html).

10. F. Guivarch voit dans « le point culminant [de] 1928-1929» une coïncidence heureuse 
avec « le centième anniversaire du premier départ de l'initiateur », tandis que J.-J. Moncus considère l'année 1929 comme l' "Âge d'or des Johnnies ». Aucun de ces deux auteurs ne dévoile malheureusement les sources documentaires qui sous-tendent leurs affirmations respectives.

11. Le singulier bretonnisé « Johnnig » rencontré dans le titre des quatre premiers épisodes ( ${ }^{9} 30$ nov. 1929) est remplacé dans le titre des onze épisodes suivants (14 déc. 1929-22 févr. 1930) par la forme non acclimatée " Johnny », dont fait également usage le corps du feuilleton, conformément à la pratique attestée chez les marchands d'oignons léonards, que suivront Naïg Rozmor ou le père Médard : le pluriel JOHNNIGED correspond au singulier JOHNNY, le phonème intercalaire $/ \mathrm{g} /$ résultant vraisemblablement d'un processus mécanique d'épenthèse. (Voir par exemple la complainte "Pôtred BroZaoz », écrite en 1927 par le « barde » saint-politain Francis Moal lors d'une « saison d'oignons » en Écosse, qui associe le pluriel « Jonnyget » au singulier « Jonnhy ».)

12. La séquence nominale «Trubuilhou eur Johnnig/y » me parait s'inspirer de l'intertitre «Trubuliou eur marc'hadour legumach e Southampton », qui coiffait un chapitre du feuilleton «Avanturiou an Aotrou Skrabelaou » de l'écrivain-colporteur léonard Loeiz ar Floc'h ( $A r$ Bobl, 15 oct. 1910). La comparaison des deux intitulés permet de mesurer l'économie linguistique que réalise en contexte breton le vocable JOHNNY, synthétisant en un mot unique et bref l'ensemble des traits sémantiques \{marchand + \{de légumes/ oignons $\}+\{$ outre-Manche $\}$.

13. Ce titre hybride à tiroirs : «Good onions, very cheap (véré chip)/(ognon mat, marc'hadmat) / GWERZ AR JOHNIGET (da gana en eur vont en hent) » se verra modifié à chaque réédition du chant, s'adaptant aux besoins ou attentes spécifiques du public visé et raccourcissant à mesure que grandira la notoriété de la chanson, e.g. : " Good onions, very scheap (véré chip) / (ognon mat, marc'had-mat) / Kanaouen nevez ar gwerzerien ognon e Bro-Zaoz » (feuille volante, Morlaix, Boclé, non dat.), " Good onions / Son ar Johniged e Rosko » (dans le recueil Mojennou ha Soniou, Rennes, Imprimerie Centrale de Bretagne, non dat., p. 111), «Good Onions » (dans la revue Bleun-Brug, n 177, maijuin 1969, p. 42).

14. Le service télévisuel régulier de la BBC venait d'être lancé en novembre 1936. 